\title{
CIRCLE PACKING COMPLEX EARTHQUAKES
}

\author{
Roger W. Barnard, Eric M. Murphy and G. Brock Williams \\ Texas Tech University, Department of Mathematics \\ Lubbock, Texas 79409, U.S.A.; roger.w.barnard@ttu.edu \\ Joint Staff Pentagon \\ Washington, DC 20318, U.S.A.; eric.murphy@js.pentagon.mil \\ Texas Tech University, Department of Mathematics \\ Lubbock, Texas 79409, U.S.A.; brock.williams@ttu.edu
}

\begin{abstract}
Thurston developed shearing earthquake maps as transformations in the Teichmüller space of hyperbolic Riemann surfaces, and McMullen expanded these transformations to include grafting earthquake maps on hyperbolic surfaces. Subsequently, the authors defined an extension of these transformations in describing complex earthquake maps on the Teichmüller space of compact tori. In this paper, we develop computational methods for these transformations using the techniques of circle packing.
\end{abstract}

\section{Introduction}

The concept of shearing maps as generalizations of Dehn twists and transformations on points in Teichmüller space was first developed by Thurston [28, 30], and has been used by Kerckhoff in proving the Nielsen Realization Conjecture [18] and Bonahon in his investigations into Thurston's boundary of Teichmüller space [6]. These earthquakes were extended by McMullen [20] to include another "geologic" action, that of grafting, on points in Teichmüller space. Taken together, through composition, we have transformations referred to as complex earthquakes, so named since the action is precisely multiplication by a complex number in the universal cover $\mathbf{H}$ of a hyperbolic surface. The action of complex earthquake maps has been expanded to include similar actions in the Teichmüller of Euclidean surfaces, specifically compact tori [2]. In this case, the nomenclature of complex earthquakes derives from the general action of the transformation as complex addition in the universal cover.

Essentially, a shearing earthquake opens a hyperbolic or Euclidean surface along a measured geodesic lamination, shears the surface along the seam in a manner determined by the measure, and re-attaches the surface. Grafting maps, rather than shearing along the lamination, insert or remove a cylinder and re-attach the surface. In the case of hyperbolic surfaces, these grafting maps (and thus complex earthquakes in general) are computationally problematic since they do not preserve the geodesic characteristics of the measured lamination. Circle packing allows for the computation of discrete conformal approximations across the full range of earthquake actions.

A circle packing is a collection of circles with a prescribed pattern of tangencies. This "prescribed pattern", is a strictly combinatorial structure with no inherent

doi:10.5186/aasfm.2011.3629

2010 Mathematics Subject Classification: Primary 30F60, 52C26.

Key words: Circle packing, conformal maps, Teichmüller theory.

The views expressed in this article are those of the authors and do not reflect the official policy or position of the United States Air Force, Department of Defense, or the U.S. Government. 
geometry. As the circles adjust their radii, trying to meet the constraints of the pattern prescribed, a rigid geometry is realized. The interplay between the combinatorial structure and the rigid constraints inherent in the geometry of the circles provides a deep link to geometric function theory and the structure of Riemann surfaces which we will exploit in describing the relationship between circle packing and the computability of earthquake maps.

Imposing the geometry of a circle implies the existence of a metric; thus we can speak about circle packings on any surface with a metric. Consequently, we may discuss circle packing on any Riemann surface. In fact, it is known that given any reasonable pattern of tangencies there exists a unique Riemann surface which supports a circle packing having that pattern of tangencies [5]. Not all surfaces, though, support a circle packing. It has been shown by Bowers and Stephenson $[8,7]$, however, that these packable Riemann surfaces are dense in Teichmüller space. To go beyond this notion of density and enter into a deterministic discussion of how the pattern of tangencies affects the geometry of the surfaces and their circle packings can be very difficult $[5,7,8,23]$.

In the discussion that follows, we will first introduce some necessary background material regarding Theicmüller spaces and the action of earthquake maps in those spaces follwed by a development of the relevant results from the theory of circle packing. With these tools, we then turn to our main results. Using techniques first developed by Williams [31, 32] we will describe a discrete circle packing version of the complex earthquakes developed by Thurston and McMullen. This process involves opening a packing along the pattern of tangencies, shearing or grafting within the combinatorics and repacking to obtain a new surface. The computational gain here is that the circle packing action is computed along every geodesic simultaneously, so the fact that McMullen's grafting maps distort geodesics is moot. We then prove that if we have a compact hyperbolic Riemann surface $R$ and a sequence of packable surfaces $R_{k}$ converging to $R$ with mesh going to zero, then we may approximate the action of a complex earthquake on $R$ through combinatorial earthquakes on the sequence $R_{k}$. A similar result then follows readily for the approximation of complex earthquake transformations on compact tori.

\section{Real and complex earthquakes}

2.1. Riemann surfaces. Our eventual goal is to define, discuss the properties of, and approximate the action of earthquakes, both grafting and shearing maps, on the Teichmüller spaces of Riemann surfaces. Here we review some of the important definitions and properties of Riemann surfaces and their deformation spaces $[11,12$, $14,16,17,19,27]$.

Definition 2.1. A Riemann surface is a one complex-dimensional manifold with charts whose overlap maps are analytic. A maximal collection of charts on a Riemann surface is a conformal structure for that surface.

The space of Riemann surfaces can be described in many ways. First, we will insist that equivalent Riemann surfaces share the same conformal structure, as described above. Further, we will require that the generators of their respective fundamental groups, also called their markings, correspond. Now, a convenient description of equivalence is through equivalence classes of quasiconformal maps. We will say 
that two quasiconformal maps $f_{1}$ and $f_{2}$ defined on a fixed Riemann surface $R$ are equivalent if and only if $f_{2} \circ f_{1}^{-1}$ is homotopic to a conformal map. We now define the Teichmüller space of a Riemann surface through this equivalence relation.

Definition 2.2. Fix a Riemann surface $R$, and suppose $f_{1}$ and $f_{2}$ are maps from $R$ to Riemann surfaces $R_{1}$ and $R_{2}$, respectively. $R_{1}$ and $R_{2}$ are equivalent in the Teichmüller space of $R$ if and only if $f_{1}$ and $f_{2}$ are equivalent,and the Teichmüller space of $R$ is the set of equivalence classes thus defined.

There is now a natural metric on Teichmüller space as a function of how close to conformal (or how quasiconformal) maps which preserve the markings might be. If we fix a Riemann surface $R$ and a marking on $R$, the distance between two points $R_{1}=f_{1}(R)$ and $R_{2}=f_{2}(R)$ in the Teichmüller space of $R$ is given by

$$
d\left(R_{1}, R_{2}\right)=\frac{1}{2} \log \left(K^{*}\right),
$$

where $K^{*}$ is the infimum of the dilatation of $g_{2} \circ g_{1}^{-1}$ where $g_{1}$ and $g_{2}$ are equivalent to $f_{1}$ and $f_{2}$, respectively. This infimum is attained, by definition, by the unique Teichmüller map.

Theorem 2.3. (Teichmüller's Theorem) Between any two points $R_{1}$ and $R_{2}$ in Teichmüller space, there is a unique quasiconformal map $T$, called the Teichmüller map, of minimal dilatation. Further, $R_{1}$ and $R_{2}$ are equivalent if and only if $T$ is homotopic to a conformal map.

This result, in particular the characterization of equivalent points is a Teichmüller space, will be critical to our proof that circle packing approximations of earthquake deformations converge.

\subsection{Laminations.}

Definition 2.4. A finite geodesic lamination $\mathcal{L}$ of a Riemann surface $R$ is a collection of finitely many disjoint geodesics on $R$ which lifts to a collection of disjoint geodesics in the universal cover for $R$.

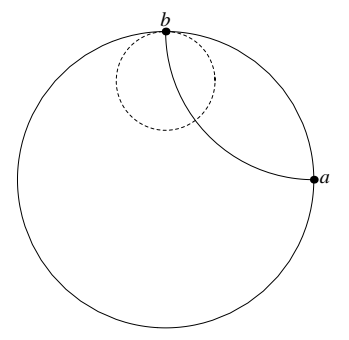

(a) Geodesic.

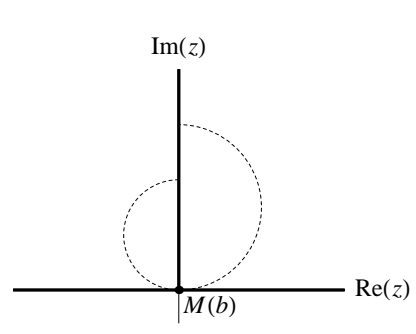

(b) "Scale" in $\mathbf{H}$.

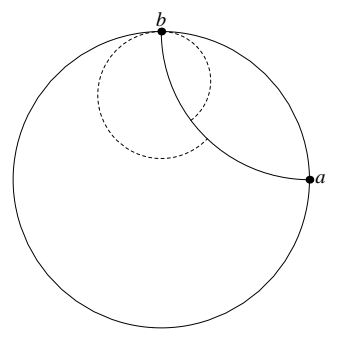

(c) Map to $\mathbf{D}$.

Figure 1. Local projective description of a shearing map.

2.3. Shearing maps. Suppose $a, b \in \partial \mathbf{D}$ such that $a \neq b$, and $L \subset \mathbf{D}$ is the unique hyperbolic geodesic connecting $a$ to $b$. Apply the Möbius transformation $M(z)$ which takes the unit disk $\mathbf{D}$ to the upper half plane $\mathbf{H}$ so that the image of $L$ is the positive imaginary axis, the image of $\partial \mathbf{D}$ is the real axis, $M(a)=\infty$, and $M(b)=0$. Now, we apply a simple scaling, $r z$, where $r>0$, in one quadrant of $\mathbf{H}$ and the identity map on the other. Finally, we apply the inverse of the Möbius transformation $M$. As we can see in Figure 1, the effect of thus composing these maps is that of 
a hyperbolic shearing. It is a simple matter to define a similar transformation on the Euclidean upper half-plane, with the multiplicative scaling action replaced by an additive translation parallel to the geodesic [2]. In this manner, we extend the shearing operation to include actions on compact tori.

2.4. Grafting maps. In geologic terms, earthquakes do not only involve lateral "shearing" along a fault line. Earthquakes may also involve a separation in which two tectonic plates move apart and new surface rises to fill the gap or a subduction in which one tectonic plate slides beneath another, and surface is lost. Just as we have maps which mimic a geologic shearing action, so we have maps - grafting mapswhich mimic these separation and subduction actions.

To define these grafting maps and imaginary earthquakes described by McMullen [20], we use an approach similar to that used for shearing maps. The difference is that rather than a piecewise scaling action given as multiplication by a real number, we apply a piecewise rotation by some angle $\alpha$ given as multiplication by the complex number $e^{i \alpha}$. This piecewise rotation leaves a gap; we fill this gap by grafting in a hyperbolic cylinder, as shown in Figure $2 \mathrm{~b}$. Now, we can map the image of the upper half plane under the maps so far applied back to the upper half plane with a power map $z^{\beta}$, where

$$
\beta=\frac{\pi}{\pi+\alpha}
$$

Taking care to define the branch cut for this power map appropriately, this map restores the real axis, and allows us to directly map the image region back to the unit disk and see the action of the earthquake on the disk. This is illustrated in Figure 2.

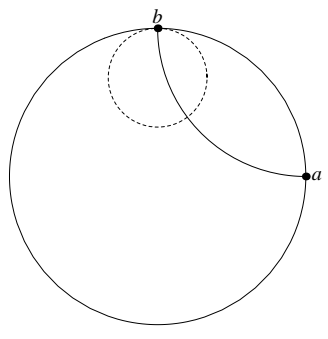

(a) Geodesic.

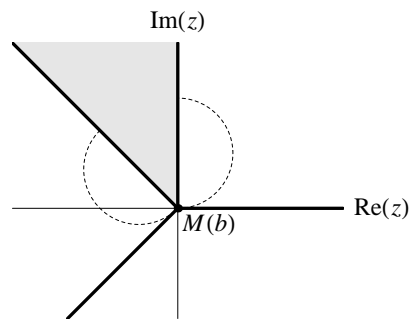

(b) "Rotate" in $\mathbf{H}$.

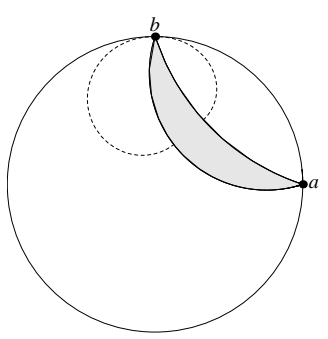

(c) Map to $\mathbf{D}$.

Figure 2. Local projective description of a grafting map.

In the application of the power map $z^{\beta}$, the arcs of circles and geodesics in Figure $2 \mathrm{~b}$ are taken to curves which are no longer either geodesics or circles. This is especially clear as we return to the unit disk $\mathbf{D}$ in Figure 2c. The two curves connecting the points $a, b \in \partial \mathbf{D}$ are clearly not geodesics, and the two disjoint halves of the dashed reference circle shown in Figure $2 \mathrm{a}$ are no longer circles. This failure to preserve geodesics and circles is key. Given a finite lamination of the disk, we may execute shearing maps on those geodesics sequentially since the maps involved are hyperbolic isometries and thus preserve the geodesics. In the case of grafting maps, however, we cannot perform the grafting actions in such a sequence, since the first application transforms the other geodesics of the lamination into curves which are no longer geodesics. 
2.5. Complex earthquakes. Now we simply define a complex earthquake as the composition of shearing and grafting maps, applied in that order. Consider the shearing and grafting maps developed in Figure 1 and Figure 2. This composition is illustrated in Figure 3.

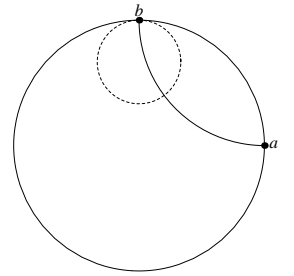

(a) Geodesic.

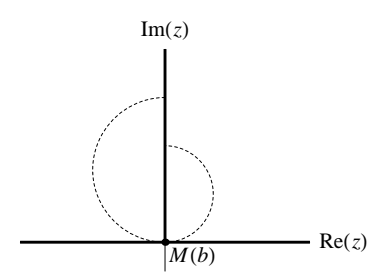

(b) "Scale" in $\mathbf{H}$.

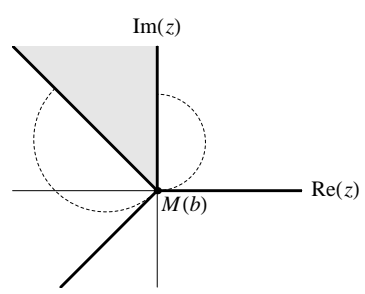

(c) "Rotate" in $\mathbf{H}$.

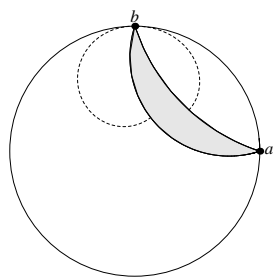

(d) Map to $\mathbf{D}$.

Figure 3. Local projective description of a complex earthquake map.

To call such a map a "complex" (as opposed to real or imaginary) earthquakes is quite natural. A real weight indicated a scaling, multiplication by a real number, and an imaginary weight indicated a rotation, multiplication by an imaginary number with unit modulus. Now, a complex weight $x+i y$ on the geodesic indicates that the transformation applied to points in one quadrant of $\mathbf{H}$ amounts to multiplication by a complex number $e^{x+i y}=e^{x} e^{i y}$. From this point, when we refer to measured geodesic laminations, we allow the measure to take complex values. We will, in the case of earthquakes on compact hyperbolic surfaces, restrict the imaginary part of complex measures to non-negative values. Otherwise, we place no a priori restriction on the weights in a measured geodesic lamination.

2.6. Earthquakes on surfaces. Now, we describe the action of earthquakes on compact Riemann surfaces with genus $g \geq 2$ (i.e., $n$-holed tori, $n \geq 2$ ) and their Teichmüller spaces. To do so, we must describe the constructions on these surfaces necessary to the earthquake actions. That is, we need measured geodesic laminations consisting of simple closed geodesics on the surface (though they will not necessarily be finite) and hyperbolic shearing and grafting maps.

Let $R$ be a compact Riemann surface with genus $g \geq 2$, (i.e., $R$ is a compact, hyperbolic Riemann surface). Just as in the disk, a finite geodesic lamination on $R$ is a finite, pairwise disjoint collection of geodesics in the intrinsic metric on $R$, but how do we define these geodesics? Well, we know that a geodesic in the Poincaré model of the hyperbolic plane is an arc of a circle which intersects $\partial \mathbf{D}$ orthogonally. Further, we know that for any hyperbolic Riemann surface, the universal cover for that surface is the hyperbolic plane where $R$ is related to the universal cover through its conformal structure $[11,12,16,17]$. So, given a curve $\gamma \subset R, \gamma$ is a geodesic on $R$ if and only if $\gamma$ lifts to a geodesic in the hyperbolic plane [25]. Thus, a lamination on the Riemann surface $R$ lifts to a geodesic lamination in the hyperbolic plane. Take a collection of simple closed geodesics in $R$, and place on each geodesic in $R$ a real (for shearing), imaginary (for grafting), or complex (for shearing then grafting) weight. This gives us a measured geodesic lamination $(\mathcal{L}, \sigma)$ on $R$. Note, however, that while we began with a finite lamination on $R$, through the lift we obtain a countably infinite lamination on $\mathbf{D}$.

Now, we can define an earthquake on $R$. A finite measured geodesic lamination on $R$ lifts to a measured geodesic lamination on $\mathbf{D}$ comprising a sequence of geodesics 
$\left\{L_{n}\right\}_{n \in \mathbf{N}}$. To this point, we have only defined finite earthquakes on the disk, so how do we define an earthquake on a countably infinite set of geodesics? We do so just as a limit of finite earthquakes.

On the surface itself, this earthquake action has a nice geometric interpretation. Shearing along a geodesic in the disk induced by a geodesic on the hyperbolic Riemann surface $R$ is equivalent to simply cutting open the surface along the geodesic, twisting by an amount prescribed by the weight on the geodesic, and gluing the two ends back together. Similarly, grafting along a geodesic in the disk induced by a geodesic on the surface $R$ is equivalent to cutting open the surface along the geodesic, inserting a hyperbolic cylinder with a height prescribed by the weight on the geodesic, and gluing the ends together.

It was shown by McMullen that the resulting object is a Riemann surface [20]. For the sake of completeness and, more importantly, to mirror the discrete case to come, we will explicitly construct a conformal structure on surfaces which have experienced an earthquake.

2.7. A conformal structure on the images of hyperbolic Riemann surfaces under complex earthquake maps. Let $R$ be a compact, hyperbolic Riemann surface, and suppose that we apply to $R$ a finite complex earthquake $E$ induced by a finite measured geodesic lamination $(\mathcal{L}, \sigma)$ on $R$, where the lamination consists of $n$ geodesics with complex weights $\sigma\left(L_{i}\right)=\mu_{i}+i \lambda_{i}, 1 \leq i \leq n$, where $\mu_{i} \in \mathbf{R}$ and $\lambda_{i} \in \mathbf{R}$ such that $\lambda_{i} \geq 0$. This earthquake thus involves both shearing maps and (positive) grafting maps composed to form the complex earthquake.

Since $R$ is a fixed Riemann surface, it has a conformal structure associated with it; say $\left\{\varphi_{v}\right\}_{v \in \Upsilon}$ is the atlas for the conformal structure on $R$. Further, since each grafting map involves the insertion of a cylinder $c_{j}$, we have a conformal structure associated with each such cylinder such that the coordinate charts map $c_{j}$ to subsets of a hyperbolic wedge. We do this by mapping first to an infinite strip and then applying the exponential map $e^{z}$ to that strip. For each of $c_{j}, 0 \leq j \leq m$, where $m$ gives the number of geodesics in the lamination $\mathcal{L}$ which have non-zero imaginary components for their weights, let $\left\{\phi_{k}^{j}\right\}_{k \in K}$ be the atlas associated with each such cylinder.

Define a pullback map $P$ on $\widehat{R}$, the image of $R$ under the complex earthquake $E$, so that the image of a point $r \in \widehat{R}$ under $P$ is the unique point (on $R$ or on one of the cylinders $c_{j}$ ) whose image under the complex earthquake $E$ is $r$.

With this information we now define a conformal structure on the Riemann surface $\widehat{R}$. To define this conformal structure we need only describe a set of coordinate charts mapping open regions in the surface $\widehat{R}$ to $\mathbf{C}$ so that the transition maps associated with the coordinate charts are analytic. It is sufficient to construct for every point $r \in \widehat{R}$ a map from an open neighborhood $U \subset \widehat{R}$ to $\mathbf{C}$ so that these maps satisfy the analyticity condition. It is sufficient to consider open sets of the following four classes.

(1) $U_{A}$ is the collection of all open sets $U_{\alpha} \subset \widehat{R}$ such that $U_{\alpha}$ does not intersect the image under the earthquake $E$ of any geodesic $L_{i}, 1 \leq i \leq n$, and is disjoint from every inserted cylinder $E\left(c_{j}\right), 1 \leq j \leq m$.

(2) $U_{B}$ is the collection of all open sets $U_{\beta} \subset \widehat{R}$ such that $U_{\beta} \subset E\left(c_{j}\right)$ for some $j, 1 \leq j \leq m$. 
(3) $U_{\Gamma}$ is the collection of all open sets $U_{\gamma} \subset \widehat{R}$ such that $U_{\gamma}$ intersects $E\left(L_{i}\right)$ for exactly one value $i, 1 \leq i \leq n$, where $\sigma\left(L_{i}\right) \in \mathbf{R}$, (i.e., the only map on that geodesic is a shearing operation).

(4) $U_{\Delta}$ is the collection of all open sets $U_{\delta} \subset \widehat{R}$ such that $U_{\delta}$ intersects both $P^{-1}(R)$ and $E\left(c_{j}\right)$ for some $j, 1 \leq j \leq m$.

Case I: Let $U_{\alpha} \in U_{A}$, and note that $P\left(U_{\alpha}\right)$ is an open set in $R$. From the conformal structure on $R$ and corresponding to this open set $P\left(U_{\alpha}\right)$ we have a coordinate chart $\varphi_{\alpha}$ such that $\varphi_{\alpha}\left(P\left(U_{\alpha}\right)\right) \subset \mathbf{H}=\{z \in \mathbf{C}: \operatorname{Im}(z)>0\}$. We thus define a coordinate chart $\psi_{\alpha}: \widehat{R} \rightarrow \mathbf{C}$ on the open set $U_{\alpha}$ by $\psi_{\alpha}=\varphi_{\alpha} \circ P$. In this way we define a family of coordinate charts $\Psi_{A}$ on $\widehat{R}$ by $\Psi_{A}=\left\{\psi_{\alpha}\right\}_{\alpha \in A}$.

Case II: Let $U_{\beta} \in U_{B}$. In this case, $U_{\beta} \subset \operatorname{Int}\left(E\left(c_{j}\right)\right)$, for some $1 \leq j \leq m$, and the pullback map $P$ takes this set to an open set on the finite cylinder $c_{j}$. Now, from the conformal structure on $c_{j}$ we have a coordinate chart $\phi_{\beta}^{j}$ corresponding to $P\left(U_{\beta}\right)$ such that $\phi_{\beta}^{j}\left(P\left(U_{\beta}\right)\right)$ is a subset of an infinite hyperbolic strip of uniform width determined by the complex part of the weight on the appropriate geodesic in $\mathcal{L}$. We then have a grafting map $g_{\beta}^{j}$ that opens the upper half-plane along the imaginary axis and glues in the hyperbolic strip. Following this with the conformal map $M_{j}$ that transforms the result into the upper half-plane, we have a map $\psi_{\beta}: \widehat{R} \rightarrow \mathbf{C}$ on the open set $U_{\beta}$ defined by $\psi_{\beta}=M_{j} \circ g_{\beta}^{j} \circ \phi_{\beta}^{j} \circ P$. In this way we define a family of coordinate charts $\Psi_{B}$ on $\widehat{R}$ by $\Psi_{B}=\left\{\psi_{\beta}\right\}_{\beta \in B}$.

Case III: Let $U_{\gamma} \in U_{\Gamma}$. From the conformal structure on $R$ and corresponding to an open set containing $P\left(U_{\gamma}\right)$ we have a map $\varphi_{\gamma}$ such that $\varphi_{\gamma}\left(P\left(U_{\gamma}\right)\right) \subset \mathbf{H}$. Now, we have disjoint sets that differ by the application of a hyperbolic shearing map, $S_{\gamma}$. We have thus defined a coordinate chart $\psi_{\gamma}: \widehat{R} \rightarrow \mathbf{C}$ on the open set $U_{\gamma}$ by $\psi_{\gamma}=S_{\gamma} \circ \varphi_{\gamma} \circ P$. In this way, we define a family of coordinate charts $\Psi_{\Gamma}=\left\{\psi_{\gamma}\right\}_{\gamma \in \Gamma}$.

Case IV: Let $U_{\delta} \in U_{\Delta}$, and note that $P\left(U_{\delta}\right)$ is divided between two distinct Riemann surfaces, $c_{j}$ and $R$. To define coordinate charts on sets of the form $U_{\delta}$ we define a pair of maps from these surfaces to the upper half-plane $\mathbf{H}$. Beginning with the cylinder, we have a map $\phi_{\delta}^{j}$ such that $\phi_{\delta}^{j}\left(P\left(E\left(c_{j}\right) \cap U_{\delta}\right)\right)$ is a hyperbolic strip of uniform width determined by the complex part of the weight on the appropriate geodesic in $\mathcal{L}$. We then have a grafting map $g_{\delta}^{j}$ that opens the upper half-plane along the imaginary axis and glues in a hyperbolic strip. At the same time, from the conformal structure on $R$ we have a map $\varphi_{\delta}$ such that $\varphi_{\delta}\left(P\left(\left(E\left(c_{j}\right)\right)^{C} \cap U_{\delta}\right)\right) \subset$ H. Applying the grafting map $g_{\delta}^{j}$ we put the pieces back together in C. Now, we may need to shear along the hyperbolic geodesic; this will depend upon whether or not the geodesic associated with this grafting action had a weight with nonzero real part. Note that the shearing will take place along that portion of the set not intersecting $E\left(c_{j}\right)$, since we define complex earthquakes as the composition of shearing and grafting maps, in that order. Finally, we know that there exists a conformal map $M_{j}$ that takes the opened half-plane to the upper half-plane.

$$
\psi_{\delta}(r)= \begin{cases}M_{j} \circ g_{\delta}^{j} \circ \phi_{\delta}^{j} \circ P(r) & \text { if } r \in E\left(c_{j}\right) \cap U_{\delta} \subset \widehat{R}, \\ M_{j} \circ S_{\delta} \circ g_{\delta}^{j} \circ \varphi_{\delta} \circ P(r) & \text { if } r \in\left(E\left(c_{j}\right)\right)^{C} \cap U_{\delta} \subset \widehat{R} .\end{cases}
$$


This function now defines a map $\psi_{\delta}: \widehat{R} \rightarrow \mathbf{C}$ on the open set $U_{\delta}$. Taking all such functions over the set of possible sets $U_{\delta} \in U_{\Delta}$, we define a family of coordinate charts $\Psi_{\Delta}=\left\{\psi_{\delta}\right\}_{\delta \in \Delta}$.

Define a collection of open sets, $\widetilde{U}=U_{A} \cup U_{B} \cup U_{\Gamma} \cup U_{\Delta}$, and a collection of maps from $\widehat{R}$ to $\mathrm{C}, \widetilde{\Psi}=\Psi_{A} \bigcup \Psi_{B} \bigcup \Psi_{\Gamma} \bigcup \Psi_{\Delta}$. Given this collection of open sets and corresponding coordinate charts on the image $\widehat{R}$, we are now in a position to show that $\widehat{R}$ is a Riemann surface. An immediate corollary of this result is the conclusion that complex earthquakes are transformations on the Teichmüller space of compact hyperbolic Riemann surfaces.

Proposition 2.5. The transition maps in the structure $(\widetilde{U}, \widetilde{\Psi})$ are analytic.

Proof. Let $U$ be the intersection of two open sets in $\tilde{U}$. We need only consider those intersections for which $U \in \mathbf{U}$. This gives four cases for the overlap region $U$ in which we must verify the analyticity of the transition maps.

Case I: Suppose that $U \in U_{A}$. There are four possible ways in which $U$ may occur as the result of intersections of sets in $\widetilde{U}$.

Case Ia: $U=U_{\alpha_{1}} \cap U_{\alpha_{2}}$, where $U_{\alpha_{1}}, U_{\alpha_{2}} \in U_{A}$. Associated with $U_{\alpha_{1}}$ and $U_{\alpha_{2}}$ we have coordinate charts $\psi_{\alpha_{1}}, \psi_{\alpha_{2}} \in \Psi_{A}$. Say $\psi_{\alpha_{1}}=\varphi_{\alpha_{1}} \circ P$ and $\psi_{\alpha_{2}}=\varphi_{\alpha_{2}} \circ P$. Now, the transition map $\psi_{\alpha_{2}} \circ \psi_{\alpha_{1}}^{-1}$ is analytic, since

$$
\psi_{\alpha_{2}} \circ \psi_{\alpha_{1}}^{-1}=\left(\varphi_{\alpha_{2}} \circ P\right) \circ\left(\varphi_{\alpha_{1}} \circ P\right)^{-1}=\varphi_{\alpha_{2}} \circ \varphi_{\alpha_{1}}^{-1}
$$

and $\varphi_{\alpha_{1}}$ and $\varphi_{\alpha_{2}}$ were taken from the conformal structure on $R$. Similarly, we know that $\psi_{\alpha_{1}} \circ \psi_{\alpha_{2}}^{-1}$ is also analytic.

The remaining sub-cases of Case I cover each of the possible forms taken by the open set $U$. In each, the approach to demonstrating analyticity is almost precisely parallel to that for Case Ia. The differences arise from differences in the conformal structures as they are defined across the various shearing and grafting geodesics and cylinders either inserted or removed. The critical element in each remaining case, however, is that the transition maps are defined only on the intersection $U$. Since the set $U$ does not itself intersect a geodesic associated with a shearing map, a geodesic associated with the insertion of a cylinder (or the boundary along which we weld/remove the cylinder to/from the Riemann surface $R$ via a grafting map), the shearing map (or other map drawn from the appropriate conformal structure) acts as either the identity or as a hyperbolic isometry. In either case, the complicating action(s) introduced in the remaining cases is/are (locally) analytic on $U$.

Case II: Suppose that $U \in U_{B}$. There are two ways in which $U$ may occur as the result of intersections of sets in $\widetilde{U}$.

Case IIa: $U=U_{\beta_{1}} \cap U_{\beta_{2}}$, where $U_{\beta_{1}}, U_{\beta_{2}} \in U_{B}$. Associated with $U_{\beta_{1}}$ and $U_{\beta_{2}}$ we have coordinate charts $\psi_{\beta_{1}}, \psi_{\beta_{2}} \in \Psi_{B}$. Say $\psi_{\beta_{1}}=M_{j} \circ g_{\beta_{1}}^{j} \circ \phi_{\beta_{1}}^{j} \circ P$ and $\psi_{\beta_{2}}=$ $M_{j} \circ g_{\beta_{2}}^{j} \circ \phi_{\beta_{2}}^{j} \circ P$. Note that since both $U_{\beta_{1}}$ and $U_{\beta_{2}}$ must necessarily lie in the same finite inserted cylinder, the grafting maps $g_{\beta_{1}}^{j}$ and $g_{\beta_{2}}^{j}$, are the same map. Now, consider the transition maps $\psi_{\beta_{2}} \circ \psi_{\beta_{1}}^{-1}$ and $\psi_{\beta_{1}} \circ \psi_{\beta_{2}}^{-1}$.

$$
\begin{aligned}
\psi_{\beta_{2}} \circ \psi_{\beta_{1}}^{-1} & =\left(M_{j} \circ g_{\beta_{2}}^{j} \circ \phi_{\beta_{2}}^{j} \circ P\right) \circ\left(M_{j} \circ g_{\beta_{1}}^{j} \circ \phi_{\beta_{1}}^{j} \circ P\right)^{-1} \\
& =M_{j} \circ g_{\beta_{2}}^{j} \circ\left(\phi_{\beta_{2}}^{j} \circ\left(\phi_{\beta_{1}}^{j}\right)^{-1}\right) \circ\left(g_{\beta_{1}}^{j}\right)^{-1} \circ M_{j}^{-1} .
\end{aligned}
$$


Now, notice that the maps $M_{j}$ and $M_{j}^{-1}$ are analytic. Next, notice that the maps $g_{\beta_{1}}^{j}$ and $g_{\beta_{2}}^{j}$ have no effect on the analyticity of the maps involved, since both $U_{\beta_{1}}$ and $U_{\beta_{2}}$ are on the interior of the cylinder $E\left(c_{j}\right)$. These maps merely indicate that we consider the infinite hyperbolic strip in a different context. The action affecting analyticity takes place in the conformal structure on the cylinder $c_{j}$, and $\phi_{\beta_{2}}^{j} \circ\left(\phi_{\beta_{1}}^{j}\right)^{-1}$ is analytic since these maps were taken from this conformal structure. Thus, as a composition of analytic maps, $\psi_{\beta_{2}} \circ \psi_{\beta_{1}}^{-1}$ is analytic. Similarly, $\psi_{\beta_{1}} \circ \psi_{\beta_{2}}^{-1}$ is analytic.

Case IIb: $U=U_{\beta} \bigcap U_{\delta}$, where $U_{\beta} \in U_{B}$ and $U_{\delta} \in U_{\Delta}$. Associated with each of $U_{\beta}$ and $U_{\delta}$ we have coordinate charts $\psi_{\beta} \in \Psi_{B}$ and $\psi_{\delta} \in \Psi_{\Delta}$, respectively. Say $\psi_{\beta}=M_{j} \circ g_{\beta}^{j} \circ \phi_{\beta}^{j} \circ P$ and $\psi_{\delta}$ of the form given in (1). Note that since $U$ does not intersect any geodesic associated with the grafting of the cylinder, for every $r \in U$ we have $r \in E\left(c_{j}\right) \cap U_{\delta} \subset \widehat{R}$, where $j$ denotes the cylinder associated with the grafting. Thus, on $U$ we have $\psi_{\delta}=M_{j} \circ g_{\delta}^{j} \circ \phi_{\delta}^{j} \circ P$. Now, consider the transition maps $\psi_{\beta} \circ \psi_{\delta}^{-1}$ and $\psi_{\delta} \circ \psi_{\beta}^{-1}$.

$$
\psi_{\beta} \circ \psi_{\delta}^{-1}=\left(M_{j} \circ g_{\beta}^{j} \circ \phi_{\beta}^{j} \circ P\right) \circ\left(M_{j} \circ g_{\delta}^{j} \circ \phi_{\delta}^{j} \circ P\right)^{-1} .
$$

This case, thus reduces to Case IIa, and the transition maps $\psi_{\beta} \circ \psi_{\delta}^{-1}$ and $\psi_{\delta} \circ \psi_{\beta}^{-1}$ are analytic.

Case III: Suppose that $U \in U_{\Gamma}$. Then, $U=U_{\gamma_{1}} \cap U_{\gamma_{2}}$, where $U_{\gamma_{1}}, U_{\gamma_{2}} \in U_{\Gamma}$. Associated with each of $U_{\gamma_{1}}$ and $U_{\gamma_{2}}$ we have coordinate charts $\psi_{\gamma_{1}}, \psi_{\gamma_{2}} \in \Psi_{\Gamma}$. Say $\psi_{\gamma_{1}}=S_{\gamma} \circ \varphi_{\gamma_{1}} \circ P$ and $\psi_{\gamma_{2}}=S_{\gamma} \circ \varphi_{\gamma_{2}} \circ P$. Note the earthquake (shearing) map in each of these coordinate charts is the same, since this earthquake is defined by intersection with a particular weighted geodesic rather than a particular set $U$. Now, consider the transition maps $\psi_{\gamma_{2}} \circ \psi_{\gamma_{1}}^{-1}$ and $\psi_{\gamma_{1}} \circ \psi_{\gamma_{2}}^{-1}$.

$$
\psi_{\gamma_{2}} \circ \psi_{\gamma_{1}}^{-1}=\left(S_{\gamma} \circ \varphi_{\gamma_{2}} \circ P\right) \circ\left(S_{\gamma} \circ \varphi_{\gamma_{1}} \circ P\right)^{-1}=S_{\gamma} \circ \varphi_{\gamma_{2}} \circ \varphi_{\gamma_{1}}^{-1} \circ S_{\gamma}^{-1}
$$

The action of the transition map here reduces to a hyperbolic isometry, and is thus analytic.

Case IV: Suppose that $U \in U_{\Delta} . U=U_{\delta_{1}} \bigcap U_{\delta_{2}}$, where $U_{\delta_{1}}, U_{\delta_{2}} \in U_{\Delta}$. Associated with each of $U_{\delta_{1}}$ and $U_{\delta_{2}}$ we have coordinate charts $\psi_{\delta_{1}}, \psi_{\delta_{2}} \in \Psi_{\Delta}$. Say $\psi_{\delta_{1}}$ and $\psi_{\delta_{2}}$ are each of the form given in (1). The set $U$ can now be decomposed into three disjoint sets, $U=U_{\alpha} \bigcup L \bigcup U_{\beta}$, where $U_{\alpha} \in U_{A}, U_{\beta} \in U_{B}$, and $L=\partial U_{\alpha} \bigcap \partial U_{\beta}$. We have already shown that the transition maps $\psi_{\delta_{1}} \circ \psi_{\delta_{2}}^{-1}$ and $\psi_{\delta_{2}} \circ \psi_{\delta_{1}}^{-1}$ are analytic on each of the sets $U_{\alpha}$ and $U_{\beta}$. These transition maps, though, are also clearly continuous on all of $U$. Since the image of $L \subset U$ in the plane has measure zero, any map which is $K$-quasiconformal on the image of $U \backslash L$ is also $K$-quasiconformal on $L$ [19]. Thus, since the transition maps are analytic (1-quasiconformal) on the images of $U_{\alpha}$ and $U_{\beta}$, the transition maps are analytic on all of $U$.

Thus, the transition maps in the structure $(\widetilde{U}, \widetilde{\Psi})$ are analytic.

Since the structure defined above describes a cover for the surface $\widehat{R}$ and a set of maps on that cover so that the transition maps are analytic, we have defined a sufficient conformal structure on the surface $\widehat{R}$; we simply include any other sets and maps compatible with this structure. A similar result for compact tori is given in [2]. 


\section{Circle packing}

3.1. Preliminaries and definitions. A circle packing is a configuration of circle with a prescribed pattern of tangencies. Thurston conjectured in 1985 that these circle packings might be used to approximate the action of conformal maps [29]. These circle packings have since been widely studied, with applications in many different areas of mathematics. We begin here with some basic definitions and a general discussion of circle packing. Several excellent resources are available with much greater detail [13, 23, 26].

Definition 3.1. A bounded degree abstract triangulation $\mathcal{K}$ is an abstract simplicial 2-complex which triangulates an orientable topological surface such that

(1) the set of interior vertices is non-empty and edge-connected;

(2) no interior edge in $\mathcal{K}$ has both vertices on the boundary;

(3) no vertex in $\mathcal{K}$ belongs to more than two boundary edges;

(4) there is an upper bound on the degree of vertices in $\mathcal{K}$.

It is this combinatorial object, the abstract triangulation, which encapsulates the "prescribed pattern of tangencies" in our circle packing. We refer to these triangulations as abstract to emphasize the fact that in the definition we have implied no concrete geometric realization. A 2-complex and, by extension, the associated abstract triangulation are purely combinatorial objects; they have no inherent geometric structure until they are realized as a circle packing.

Definition 3.2. A circle packing is a configuration of circles with a specified pattern of tangencies. In particular, if $\mathcal{K}$ is an abstract triangulation of a topological surface, then a circle packing $P$ for $\mathcal{K}$ is a configuration of circles such that

(1) $P$ contains a circle $C_{v}$ for every vertex $v \in \mathcal{K}$;

(2) if $[u, v]$ is an edge of $\mathcal{K}$, then $C_{v}$ is externally tangent to $C_{u}$;

(3) if $\langle v, u, w\rangle$ is a positively oriented face of $\mathcal{K}$, then $\left\langle C_{v}, C_{u}, C_{w}\right\rangle$ forms a positively oriented mutually tangent triple of circles in $P$.

A circle packing is called univalent if the circles in the packing have mutually disjoint interiors. That is, the packing is univalent if no two circles intersect in more than one point. This univalent circle packing represents a geometric realization of the underlying abstract triangulation $\mathcal{K}$. Vertices in the triangulation may be realized in this packing as the centers (in some particular geometry, hyperbolic, Euclidean, or spherical) of the circles, and the edges as geodesic segments connecting the centers. This embedding is called the carrier of the circle packing.

If $\mathcal{K}$ is embedded in $\mathbf{C}$ in two different ways there is a natural piecewise affine map from the carrier associated with one packing to the other achieved by sending triangles of one packing to their counterparts in the other packing. These piecewise affine maps are referred to as discrete conformal maps.

3.2. Discrete function theory. The important characteristic of the discrete conformal maps induced by circle packings is not that they are quasiconformal; the key fact, suggested by Thurston [30] and proven by Rodin and Sullivan [22], is that these maps are "nearly conformal." This is the result given in Theorem 3.6, the Rodin-Sullivan Theorem. Before we state this theorem, however, we first state some geometric results associated with circle packing that are interesting in themselves and required for the proof of Theorem 3.6. 
Lemma 3.3. (Length-Area Lemma) Let $P$ be a univalent packing in $\mathbf{D}$ and $C_{v}$ a circle in $P$ with Euclidian radius $r$. Assume there exist $m$ disjoint chains of circles in $P$ having combinatorial lengths $n_{1}, n_{2}, \ldots, n_{m}$, such that each chain separates $C_{v}$ from 0 and a point on $\partial \mathbf{D}$. Then

$$
r<\frac{4}{\sqrt{\sum_{i=1}^{n} \frac{1}{n_{i}}}} .
$$

As the number of generations separating a circle in a packing from the boundary increases Lemma 3.3, the Length-Area Lemma, has the effect of forcing the radius of this circle to zero (in the limit). For a more detailed discussion of Lemma 3.3, the Length-Area Lemma, see [22, 23].

Lemma 3.4. (Ring Lemma) Given a univalent flower $\left(C_{v_{0}} ; C_{v_{1}}, C_{v_{2}}, \ldots, C_{v_{n}}\right)$ in $\mathrm{C}$ there is a lower bound $C_{n}$, depending only on $n$, on the ratio of the radius $r_{i}$ of $C_{v_{i}}$ to the radius $r_{0}$ of $C_{v_{0}}$ for each $i=1,2, \ldots, n$; that is

$$
C_{n}<\frac{r_{i}}{r_{0}}
$$

$i=1,2, \ldots, n$.

The Ring Lemma, guarantees that central angles in the carrier on a flower are bounded away from zero and $\pi$. That is, suppose we are given a complex $\mathcal{K}$ in which the degree of each vertex, the number of adjacent vertices, is bounded; also suppose we have two (different) packings $P_{1}$ and $P_{2}$ associated with $\mathcal{K}$. The Ring Lemma guarantees that the quasiconformality of the induced conformal map from $P_{1}$ to $P_{2}$ is bounded.

Lemma 3.5. (Hexagonal Packing Lemma) There is a sequence $\left\{s_{n}\right\}_{n} \in \mathbf{N}$, decreasing to zero, with the following property. Let $c_{1}$ be a circle in a univalent Euclidean circle packing $P$, and suppose the first $n$ generations of circles about $c_{1}$ are combinatorially equivalent to $n$ generations of the regular hexagonal packing around one of its circles. Then for any circle $c \in P$ tangent to $c_{1}$,

$$
\left|1-\frac{r_{c}}{r_{c_{1}}}\right| \leq s_{n},
$$

where $r_{c}$ is the radius of the circle $c$ in $P$ and $r_{c_{1}}$ is the radius of the circle $c_{1}$ in $P$.

The immediate value of Lemma 3.3, Lemma 3.4, and Lemma 3.5 is their use in proving Theorem 3.6, the Rodin-Sullivan Theorem, one of the fundamental results in the study of circle packing.

Theorem 3.6. (Rodin-Sullivan Theorem) Fix a simply connected domain $\Omega \subsetneq$ $\mathrm{C}$ and points $p, q \in \Omega$. Let $P_{k}$ be the portion lying in $\Omega$ of the infinite regular hexagonal packing whose circles all have radius $\frac{1}{k}$, and let $\mathcal{K}_{k}$ be the underlying complex for the packing $P_{k}$. Suppose $\widetilde{P}_{k}$ is a packing in $\mathbf{D}$ for $\mathcal{K}_{k}$ with all boundary circles tangent to $\partial \mathbf{D}$, and let $f_{k}: \operatorname{carr}\left(P_{k}\right) \rightarrow \operatorname{carr}\left(\widetilde{P}_{k}\right)$ be the induced discrete conformal map. If each $\widetilde{P}_{k}$ has been normalized so that $f_{k}(p)=0$ and $f_{k}(q)>0$, then $\left\{f_{k}\right\}$ converges locally uniformly to the unique Riemann map $f: \Omega \rightarrow \mathbf{D}$ satisfying $f(p)=0$ and $f(q)>0$. 
The requirement that each packing in the Rodin-Sullivan Theorem be uniformly degree six is quite restrictive. Since the initial proof, however, Stephenson [24] relaxed the degree six condition using techniques of random walks, and He and Rodin [15] showed that only a uniform bound on the degree is necessary. To thus relax the requirement on the combinatorics of the packing, we require Lemma 3.7, sometimes referred to as the Packing Lemma.

Lemma 3.7. (Packing Lemma) Let $\left\{\mathcal{K}_{n}\right\}_{n \in \mathbf{N}}$ be a sequence of combinatorial closed disks such that

(1) there exists a uniform bound on the degree of the vertices in $\mathcal{K}_{n}$ for each $n \in \mathbf{N}$, and

(2) the sequence $\left\{\mathcal{K}_{n}\right\}_{n \in \mathbf{N}}$ is either a nested sequence which exhausts a parabolic combinatorial disk or is asymptotically parabolic.

There exists a sequence $\left\{s_{m}\right\}_{m \in \mathbf{N}} \subset \mathbf{R}$, decreasing to zero, with the following property. Suppose that for some $n, u$ and $v$ are adjacent interior vertices of $K_{n}$ whose combinatorial distance from $\partial \mathcal{K}_{n}$ are both at least $m$, and suppose that $P_{n}$ and $\widetilde{P}_{n}$ are two univalent, Euclidean circle packings for $\mathcal{K}_{n}$. Then

$$
\left|\frac{\tilde{r}_{u}}{\tilde{r}_{v}}-\frac{r_{u}}{r_{v}}\right| \leq s_{m}
$$

where $r_{u}$ and $r_{v}$ are the radii of the circle in $P_{n}$ corresponding to $u$ and $v$, and $\tilde{r}_{u}$ and $\tilde{r}_{v}$ are the radii of the circle in $\tilde{P}_{n}$ corresponding to $u$ and $v$.

Essentially, the Packing Lemma states that for a circle "deep" in a packing, the ratio of its radius to any given neighbor is nearly the same in the packings $P_{n}$ and $\widetilde{P}_{n}$; in other words, the triangles in $P_{n}$ and $\widetilde{P}_{n}$ are nearly similar triangles. This gives us that away from the boundary, the induced discrete conformal map between $P_{n}$ and $\widetilde{P}_{n}$ is nearly conformal. This fact will play an important role in the proof of our main result in Section 4.

3.3. Hex refinement. In order to obtain the various approximation results for circle packing and discrete analytic function theory, we require a method to refine given circle packings. The primary requirement in any such refinement is to maintain some uniform control over the degree of the complexes generated by the refinement algorithm since we need the Ring Lemma to apply at each successive level of refinement. The hex refinement method developed by Bowers and Stephenson [9] is especially nice.

If $\mathcal{K}$ is a 2-complex, the hex refinement of $\mathcal{K}$ is the complex formed by adding a vertex to each edge and adding an edge between any two vertices lying on the same face. Note that hex refinement, and refinement in general, is really a combinatorial process, refining the combinatorics of the complex $\mathcal{K}$; one must repack the new complex obtained by refining $\mathcal{K}$ in order to realize the effect of the refinement in a circle packing.

Further, we notice that refining only one edge in a complex is not permitted, since this would result in a complex that is not a triangulation; the faces bordering the refined face will have an extra vertex along the common edge they share with the refined face, giving combinatorial quadrilaterals rather than triangles. We can, however, locally refine only those triangles in the complex which present some difficulty with respect to desired characteristics of the complex, then correct the introduced 
problems on adjacent faces by adding a single edge from a vertex to the midpoint of the opposite side.

This process of hex refinement of individual faces (or subsets of the triangles in a triangulation) and the addition of edges to absorb extra vertices can be used to locally refine an abstract triangulation in order to improve the discrete approximation in troublesome areas. This property of local refinement will play a key role in the discrete conformal approximation of earthquakes in Section 4.

3.4. Combinatorial welding. We now describe the process of combinatorial welding developed by Williams [31, 32]. Suppose two abstract triangulations $\mathcal{K}_{1}$ and $\mathcal{K}_{2}$ are embedded in a surface $S$ and suppose $h: B_{1} \subset \partial \mathcal{K}_{1} \rightarrow B_{2} \subset \partial \mathcal{K}_{2}$ is a homeomorphism. The map $h$ will be used to attach the triangulations $\mathcal{K}_{1}$ and $\mathcal{K}_{2}$ along the subsets of their respective boundaries $B_{1}$ and $B_{2}$ and form a new triangulation $\mathcal{K}$. In Section $4, h$ will be taken as a discrete version of the map defining an earthquake along a geodesic, and the attaching described here will act as a combinatorial shearing and/or combinatorial grafting in the construction of combinatorial earthquakes.

If the map $h$ respects the combinatorial structures of $\mathcal{K}_{1}$ and $\mathcal{K}_{2}$ (i.e., if $h$ sends vertices and edges in $B_{1}$ and $B_{2}$ to vertices and edges, respectively) the combinatorial welding process is trivial. We simply identify vertices and edges in $B_{1} \subset \partial \mathcal{K}_{1}$ with their images in $B_{2} \subset \partial \mathcal{K}_{2}$, as shown in Figure 4a. Note that the action of $h$ on the vertices in $B_{1}$ is sufficient to determine the combinatorial action of $h$ on all of $B_{1}$.

More interesting is the case in which $h$ does not respect the combinatorics of $\mathcal{K}_{1}$ and $\mathcal{K}_{2}$ in this way. In general, the images of vertices in $B_{1}$ under that action of $h$ will not be vertices in $B_{2}$. To ensure the map $h$ is well-defined in these cases, it is necessary to modify the triangulations $\mathcal{K}_{1}$ and $\mathcal{K}_{2}$ so that $h$ respects the modified combinatorial structure. This modification is accomplished using a refinement similar to the hex refinement procedure discussed above. Specifically, for each boundary vertex $v \in B_{1}$ we refine $B_{2}$ by adding a vertex to $B_{2}$ embedded at the point $h(v)$. Likewise, for each boundary vertex $w \in B_{2}$ we refine $B_{1}$ by adding a vertex to $B_{1}$ embedded at the point $h^{-1}(w)$. When vertices are thus inserted, however, $\mathcal{K}_{1}$ and $\mathcal{K}_{2}$ will not necessarily remain triangulations. To maintain the characteristics of a triangulation, we further refine each of $\mathcal{K}_{1}$ and $\mathcal{K}_{2}$. If we add a vertex $h(v)$ or $h^{-1}(w)$ to an edge $[a, b]$ of a triangle $\langle a, b, c\rangle$, we refine the original complex (of $\mathcal{K}_{1}$ or $\mathcal{K}_{2}$ ) by adding an edge from $c$ to the new vertex. An example of this process is shown in Figure 4b. This refinement process is well-defined and produces new triangulations since, by definition, each face in the triangulations may have at most one edge contained in the boundary. We therefore add vertices to only one edge in any given face which might interfere with the refinement process.

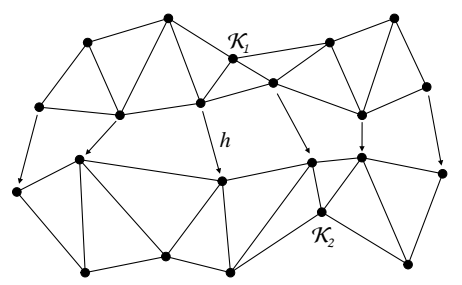

(a) Welding action when $h$ respects the combinatorics of $\mathcal{K}_{1}$ and $\mathcal{K}_{2}$.

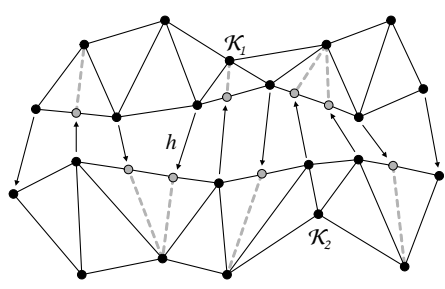

(b) Welding action with combinatorial refinement of $\mathcal{K}_{1}$ and $\mathcal{K}_{2}$.

Figure 4. Combinatorial welding and refinement. 
It is important that in the course of executing these combinatorial weldings and then embedding the resulting combinatorics in a circle packing to realize a geometry that we maintain control over the combinatorial and geometric characteristics of the process. Specifically, we must ensure

(1) the degree of the complex resulting from the combinatorial welding is bounded, and

(2) the new edges added to the carriers of $\mathcal{K}_{1}$ and $\mathcal{K}_{2}$ do not result in triangles with arbitrarily small angles.

Condition (1) will then guarantee that we can apply Lemma 3.4, the Ring Lemma, to the packing associated with the new complex which results from the combinatorial welding, thus giving bounds on the angles in the new packing. Condition (2) will ensure the existence of similar bounds for the packing prior to welding and repacking; when we artificially insert edges in the carrier of the initial packing so that there is a bijection between triangles in the two carriers, we must not create triangles with arbitrarily small angles. Thus, with these two conditions satisfied we may construct quasiconformal discrete maps between the carriers in the surfaces associated with the initial packing and the post-welding packing. These technical considerations are easily overcome through the use of local hex refinement (Condition (1)) and through "rounding off" the refinement to existing vertices if the new vertex added in the welding refinement is too close to an existing vertex (Condition (2)). The details of these processes, and verification of their validity, are given in [31, 32].

Once we have welded the two sides of the complex together in this way, we may pack the resulting complex. Now, we have a bijection between the triangles in the carrier of the original packing (after it has been suitably refined) and the new packing which results once the shearing operation is completed; we use these triangles to construct a piecewise affine, and thus quasiconformal, map from the original packing to the new packing. Thus, the discretization of the map along the boundary leads to a discretization of the map on the entire surface. This now leads us to Proposition 3.8, proven in [32].

Proposition 3.8. Combinatorial attachment by $h$ induces a piecewise linearization $\hat{h}$ of $h$. If $h$ is bilipschitz, then the linearization $\hat{h}$ will be bilipschitz as well.

3.5. Density of packable surfaces. Demonstrations of the existence of circle packings have been given variously by Thurston [30], Minda and Rodin [21], and Beardon and Stephenson [5]. Brooks [10] showed that compact packable surfaces are dense in moduli space, and Bowers and Stephenson [7, 8] extended this result to include surfaces of finite analytic type. Other results on the density of packable surfaces have been given by Barnard and Williams [3] and Williams [32]. The results thus demonstrated which are germane here are summarized below.

Theorem 3.9. Let $\mathcal{K}$ be an abstract triangulation of a surface of type $(g, n, m)$. There exists a unique surface in moduli space which supports a packing for $\mathcal{K}$. A complex, along with a choice of marking, then determines a unique point in Teichmüller space. Moreover, the collection of all packable surfaces is dense in Teichmüller space.

The density of merely packable surfaces as given here is extremely useful and powerful. Of equal interest, however, is the density of other subsets of packable surfaces; we introduce class of compact Riemann surfaces called equilateral surfaces 
which will be useful in constructing sequences of packable Riemann surfaces while maintaining control over the combinatorics of their underlying complexes.

Definition 3.10. Suppose $S$ denotes a compact, orientable topological surface and let $\mathcal{K}$ denote a triangulation of the surface $S$. If we paste together equilateral triangles (triangles conformally equivalent to equilateral triangles) in the pattern of $\mathcal{K}$ to impose a piecewise affine structure on $S$, this affine structure defines a conformal structure on $S$, guaranteeing that $S$ is a Riemann surface. Riemann surfaces thus constructed are called equilateral surfaces.

Just as has been shown for compact surfaces by Brooks [10] and for surfaces of finite analytic type by Bowers and Stephenson [7, 8], if we fix a genus $g>0$ this seemingly very restrictive class of (compact) equilateral surfaces of genus $g$ is dense in Teichmüler space of genus $g$ [4]. We state this result as Theorem 3.11.

Theorem 3.11. (Belyı) If $S$ is a Riemann surface of genus $g>0$, the set of equilateral surfaces of genus $g$ is countable and dense in the Teichmüller space of $S$.

This result guarantees the existence of sequences of packable Riemann surfaces with underlying combinatorial structures over which we may exercise a significant degree of control. This ability to control the underlying combinatorics allows us to manipulate the geometry of our packings in that we may force the circles in a sequence of packable (and packed) surfaces to decrease in size [1].

Lemma 3.12. Let $S$ be a Riemann surface and let $R$ be an arbitrary point in the Teichmüller space of $S$. There exists a sequence of points $\left\{R_{n}\right\}$ in the Teichmüller space of $S$ such that $R_{n} \rightarrow R$ in the Teichmüller metric as $n \rightarrow \infty, R_{n}$ is packable for every $n$, and the radii of the circles in $P_{n}$ go to zero as $n \rightarrow \infty$, where $P_{n}$ is the unique packing on the surface $R_{n}$ for every $n$.

\section{Packing earthquakes}

Throughout this chapter let $(\mathcal{L}, \sigma)$ be a finite measured geodesic lamination of D associated with a set of points $S \subset \mathbf{D}$. Suppose $\mathcal{L}$ consists of $n$ disjoint, simple, closed geodesics $\left\{L^{i}\right\}_{i=1}^{n}$. Note that throughout this chapter we suppose that the real (shearing) part of the weight on any geodesic $L^{i}$ may assume any real value, while the imaginary (grafting) part may assume only non-negative real values. This indicates that the shearing maps we consider may shear to either the left or the right, and the grafting maps we consider involve only the insertion of cylinders of positive height. Much of the construction below is taken from [32]; we reproduce it here in the interest of making this exposition as self-contained as possible.

4.1. Discrete laminations. Let $\left\{P_{n}\right\}_{k=1}^{\infty}$ be a sequence of packings in $\mathbf{D}$ with uniformly bounded degree and mesh decreasing to zero. Let $D_{k}$ denote the Euclidean carrier of $P_{k}$ for each $k \in \mathbf{N}$. Note that the construction of a "combinatorial" earthquake $E_{k}$ of this Euclidean carrier as an approximation of the finite earthquake $E$ is complicated by the fact that $D_{k}$ is not equal to $\mathbf{D}$ for any fixed $k \in \mathbf{N}$.

Note that any given geodesic in the lamination $\mathcal{L}$ will pass indiscriminately through the points and triangles of the Euclidean carrier $D_{k}$. Thus, shearing and grafting $D_{k}$ along the geodesics of the lamination is not directly possible. In order to approximate the action of the earthquake $E$, therefore, we construct a combinatorial lamination of $D_{k}$ that respects the combinatorial structure and converges to the finite 
geodesic lamination $\mathcal{L}$ as $k \rightarrow \infty$. This combinatorial lamination comprises combinatorial "geodesics" $\left\{\ell_{k}^{i}\right\}_{i=1}^{n}$ corresponding to the true hyperbolic geodesics $\left\{L^{i}\right\}_{i=1}^{n}$, constructed in the following manner.

We consider packings that are hyperbolic in $\mathbf{D}$; that is, we consider packings that fill the disk. Let $a_{k}^{i}$ and $b_{k}^{i}$ be the endpoint of the geodesic $L^{i} \subset \partial \mathbf{D}$. We construct $\ell_{k}^{i}$ as an edge path which, in the limit, connects $a_{k}^{i}$ and $b_{k}^{i}$, whose Hausdorff distance to $L^{i}$ is minimal, and which satisfies the following three conditions.

(1) Every vertex in $\ell_{k}^{i}$ must have a neighbor in both components of $D_{k} \backslash \ell_{k}^{i}$.

(2) The combinatorial geodesics $\left\{\ell_{k}^{i}\right\}$ are disjoint, except possibly at their common endpoints.

(3) Any hyperbolic geodesic perpendicular to $L^{i}$ intersects $\ell_{k}^{i}$ in exactly one point.

It is not immediately clear that we can in general construct such a rigidly characterized edge path through the combinatorics of $D_{k}$. Lemma 4.1 guarantees that such constructions are, in fact, possible [32].

Lemma 4.1. By taking $k$ sufficiently large and possibly modifying $D_{n}$ near $L^{i}$, we can find paths $\ell_{k}^{i}$ so that conditions (1) through (3) are satisfied. Moreover, $\ell_{k}^{i} \rightarrow L^{i}$ uniformly as $k \rightarrow \infty$.

4.2. Hyperbolic projections. Having described a discrete analog of the finite measured geodesic lamination $(\mathcal{L}, \sigma)$, we now must describe discrete analogs for the hyperbolic shearing maps and grafting maps associated with each geodesic. We cannot, of course, use the shearing and grafting maps associated with the lamination $(\mathcal{L}, \sigma)$ directly; rather, we construct projections $p_{k}^{i}$ which take the combinatorial geodesics $\ell_{k}^{i}$ to $L^{i}$. Thus, by pre-and post-composition with the map $p_{k}^{i}$, we may construct an appropriate transformation to give a discrete shearing and/or grafting along $\ell_{k}^{i}$ using the shearing and/or grafting map on $L^{i}$.

Notice that the collection of hyperbolic geodesics perpendicular to the geodesic $L^{i}$ in the lamination fill $\mathbf{D}$. By the construction of the discrete geodesic $\ell_{k}^{i}$, each such perpendicular geodesic $\eta$ intersects $\ell_{k}^{i}$ exactly once. We may thus define a map $p_{k}^{i}: D_{k} \cap \mathbf{D} \rightarrow \mathbf{D}$ by the requirement that $\left.p_{k}^{i}\right|_{\eta}$ be the unique hyperbolic Möbius transformation with axis $\eta$ and translation length equal to the hyperbolic distance from $\ell_{k}^{i} \cap \eta$ to $L^{i} \cap \eta$. The action of such a map on a segment of a discrete geodesic in $\mathbf{D}$ corresponding to the geodesic between 1 and $i$ is clearly a quasisymmetry.

We have constructed discrete geodesic approximations $\ell_{k}^{i}$ to the geodesic $L^{i}$ and the corresponding hyperbolic projections $p_{k}^{i}$, and Lemma 4.1 guarantees that $\ell_{k}^{i} \rightarrow L^{i}$ as $k \rightarrow \infty$. We now ask how the hyperbolic projections $p_{k}^{i}$ behave as $k \rightarrow \infty$ [32].

Proposition 4.2. The sequence $\left\{p_{k}^{i}\right\}$ is uniformly bilipschitz on $\overline{\mathbf{D}}$ and converges uniformly to the identity map on $\overline{\mathbf{D}}$.

4.3. Discrete shearing and grafting maps. With the hyperbolic projections $\left\{p_{k}^{i}\right\}$ we may define discrete shearing operations $\left\{h_{k}^{i}\right\}$ which approximate the hyperbolic shearing maps $\left\{h^{i}\right\}$ such that $h_{k}^{i} \rightarrow h^{i}$ as $k \rightarrow \infty$. For each $k \in \mathbf{N}$, define $h_{k}^{i}$ by

$$
h_{k}^{i}=\left(p_{k}^{i}\right)^{-1} \circ h^{i} \circ\left(p_{k}^{i}\right) .
$$

Similarly, we may use the hyperbolic projections $\left\{p_{k}^{i}\right\}$ to define discrete grafting operations $\left\{g_{k}^{i}\right\}$ which approximate the hyperbolic grafting maps $\left\{g^{i}\right\}$ such that $g_{k}^{i} \rightarrow$ $g^{i}$ as $k \rightarrow \infty$. First, we need to understand the action of the grafting map, $g^{i}$. The 
action of this map is to open the disk along the geodesic $L^{i}$, creating two copies of the geodesic. We then parameterize each copy of the geodesic and the boundary of an infinite hyperbolic strip by arc length and identify corresponding points in the parameterizations. The only difference in the discrete grafting is that the discrete geodesic $\ell_{k}^{i}$ and $L^{i}$ do not agree. So, for each $k \in \mathbf{N}$, define $g_{k}^{i}$ by

$$
g_{k}^{i}=\left(p_{k}^{i}\right)^{-1} \circ g^{i} \circ\left(p_{k}^{i}\right) .
$$

Proposition 4.3. The discretized maps $h_{k}^{i}$ and $g_{k}^{i}$ are bilipschitz on $\ell_{k}^{i}$ with bilipschitz constants bounded independently of $i$ and $k$, and $h_{k}^{i}$ and $g_{k}^{i}$ converge uniformly to the hyperbolic grafting maps $h^{i}$ and $g^{i}$ on $\mathbf{D}$ as $k \rightarrow \infty$.

Proof. The fact that the maps $h_{k}^{i}$ and $g_{k}^{i}$ are bilipschitz on $\ell_{k}^{i}$ follows directly from a result of Williams in [32]. As for convergence, Proposition 4.2 guarantees that $p_{k}^{i}$ converges uniformly to the identity. Thus, $h_{k}^{i}$ and $g_{k}^{i}$ converge uniformly to $h^{i}$ and $g^{i}$ as $k \rightarrow \infty$.

By Proposition 4.3, our discrete analogs of hyperbolic grafting and shearing converge to those grafting and shearing maps. We now define a combinatorial complex earthquake as the composition of a discrete grafting map with a discrete shearing map, $E_{k}=g_{k} \circ h_{k}$, just as we defined continuous earthquakes as the composition of grafting and shearing maps, $E=g \circ h$.

4.4. Convergence of discrete earthquake maps on hyperbolic Riemann surfaces. Thus far, we have described a family of transformations on the Teichmüller space of a hyperbolic Riemann surface and a process whereby we may discretely mimic the action of those transformations through circle packing. In Theorem 4.4 we show that this "mimicry" is in fact a convergent numerical method.

Theorem 4.4. Let $R$ be a compact hyperbolic Riemann surface with a finite measured geodesic lamination $(\mathcal{L}, \sigma)$. Let $\left\{P_{k}\right\}$ be a sequence of finite bounded degree packings with mesh decreasing to zero corresponding to Riemann surfaces $\left\{R_{k}\right\}$ such that $R_{k} \rightarrow R$ as $k \rightarrow \infty$ in the Teichmüller metric. Then the surfaces $E_{k}\left(R_{k}\right)=\widehat{R}_{k}$ induced by the discrete earthquake maps $E_{k}$ converge to the surface $E(R)=\widehat{R}$ under the earthquake map $E$ induced by $(\mathcal{L}, \sigma)$.

Proof. Let $R$ be a compact hyperbolic Riemann surface, and let $(\mathcal{L}, \sigma)$ be a finite measured geodesic lamination on $R$. In a sense, the proof of this result reduces to showing that the (6) commutes.

$$
\begin{aligned}
& \left\{R, c_{1}, c_{2}, \ldots, c_{m}\right\} \stackrel{E}{\longrightarrow} \widehat{R} \\
& f_{k} \uparrow f_{j_{k}} \quad \uparrow \hat{f}_{k} \\
& \left\{R_{k}, c_{1_{k}}, c_{2_{k}}, \ldots, c_{m_{k}}\right\} \stackrel{E_{k}}{\longrightarrow} \widehat{R}_{k}
\end{aligned}
$$

Requiring the mesh of the sequence of bounded degree packings $\left\{P_{k}\right\}$ to approach zero as $k \rightarrow \infty$ is equivalent to requiring that the radii of the circles in the packings approach zero, and we are guaranteed the existence of such a sequence by Lemma 3.12. Further, the construction of the surfaces in the proof of Lemma 3.12 allows the freedom to control the degree of vertices adjacent to the geodesics along which we shear and graft. 
The Teichmüller distance between the packable surfaces $R_{k}$ and $R$ is going to zero, so for each $k \in \mathbf{N}$ there exists a map $f_{k}: R_{k} \rightarrow R$ that respects the markings on $R_{k}$ and $R$ and is $1+\varepsilon_{k}$-quasiconformal, where $\varepsilon_{k} \rightarrow 0$ as $k \rightarrow \infty$, (i.e., as $k \rightarrow \infty$, $f_{k} \rightarrow f$, where $f$ is conformal). More precisely, the map $f_{k}$ consists of a map from the disk (or other model of the hyperbolic plane) to the disk through a map between the surfaces and their respective conformal structures. Where no ambiguity arises, however, we will speak about the map as $f_{k}: R_{k} \rightarrow R$.

Let $\left\{c_{j}\right\}_{j=1}^{m}$ be those cylinders grafted into the Riemann surface $R$ by the finite earthquake $E$. For each $j=1,2, \ldots, m$, let $\left\{c_{j_{k}}\right\}_{k \in \mathbf{N}}$ be a sequence of cylinders admitting a circle packing of finite, uniformly bounded degree so that $c_{j_{k}} \rightarrow c_{j}$ as $k \rightarrow \infty$ in the Teichmüller metric. Further, require the mesh of the packing on $c_{j_{k}}$ to go to zero as $k \rightarrow \infty$ for each $j=1,2, \ldots, m$. Thus, for each $k \in \mathbf{N}$ there exists a map $f_{j_{k}}: c_{j_{k}} \rightarrow c_{j}$ that is $1+\varepsilon_{j_{k}}$-quasiconformal, where $\varepsilon_{j_{k}} \rightarrow 0$ as $k \rightarrow \infty$, $j=1,2, \ldots, m$, (i.e., as $k \rightarrow \infty, f_{j_{k}} \rightarrow f_{j}$, where $f_{j}$ is conformal).

Let $\widehat{R}$ be the image of $R$ under the action of the earthquake $E$, and for each $k \in \mathbf{N}$ let $\widehat{R}_{k}$ be the image of $R_{k}$ under the action of a combinatorial earthquake $E_{k}$. We construct these combinatorial earthquakes by describing laminations on the surfaces $R_{k}$ corresponding to the lamination on $R$. Since on a hyperbolic Riemann surface there is exactly one simple closed geodesic in each non-zero homotopy class $[11,16]$, corresponding to each geodesic in the lamination $\mathcal{L}$ on $R$ there is a unique geodesic on $R_{k}$ in the corresponding homotopy class. Taking the collection of these geodesics, we have a finite measured geodesic lamination $\left(\mathcal{L}_{k}, \sigma\right)$ on $R_{k}$. Notice that we have left the weights $\sigma$ unchanged; we simply assign to each geodesic in $\mathcal{L}_{k}$ the weight on the corresponding geodesic in $R$. Now, we have finite measured geodesic laminations on each $R_{k}, k \in \mathbf{N}$, and we may carry out the discrete shearing and welding operations of combinatorial earthquakes.

Around each geodesic $L^{i}, i=1,2, \ldots, n$, in the lamination $\mathcal{L}$ on $R$ we place a collar, a neighborhood of uniform width isometric to a cylinder, so that no two such neighborhoods intersect. Call this collar $C^{i}$. Since the geodesics in the finite lamination are disjoint by construction, the Collar Theorem [11] guarantees that there exists such a collar about each geodesic. Similarly, about each geodesic $L_{k}^{i}, i=$ $1,2, \ldots, n$ in the lamination $\mathcal{L}_{k}$ on $R_{k}$ there exists a collar $C_{k}^{i}$ such that the collection of collars on $R_{k}$ is disjoint. We require that the collar $C_{k}^{i}$ on $R_{k}$ have width less than $\frac{1}{2}$ the width of the corresponding $C^{i}$ on $R$. Now, since $R_{k} \rightarrow R$ in the Teichmüller metric, the maps between $R_{k}$ and $R$ are going to conformal maps and the markings on $R_{k}$ are converging to the markings on $R$. Thus, for $k$ sufficiently large, the image of the collar $C_{k}^{i}$ under the map $f_{k}$ is contained in the collar $C^{i}$, and, by construction, this containment is proper, (i.e., $f_{k}\left(C_{k}^{i}\right) \varsubsetneqq C^{i}$ for all $k$ sufficiently large). Further, since the mesh of the packings on $R_{k}$ goes to zero, for $k$ sufficiently large, the discrete geodesic $\ell_{k}^{i}$ corresponding to $L_{k}^{i}, i=1,2, \ldots, n$, lies strictly within the interior of the collar $C_{k}^{i}$.

Consider the images of the collars $C_{k}^{i}$ under the action of the discrete earthquake $E_{k}$. If the geodesic $L_{k}^{i}$ about which we built the collar $C_{k}^{i}$ has real weight, and thus induces only a discrete shearing action on the discrete geodesic, then there is no difficulty in defining what we will call a quasicollar on $\widehat{R}_{k}$ as the image of $C_{k}^{i}$ under $E_{k}$. The image of the discrete geodesic $\ell_{k}^{i}$ and the image of the geodesic $L_{k}^{i}$ are contained in this quasicollar. 
The case in which the weight on $L_{k}^{i}$ has non-zero imaginary part is somewhat more problematic. The action of the earthquake in this case will divide the collar into two disjoint sets. Since the grafting (imaginary) component of the earthquake involves the insertion of a non-trivial cylinder, however, we may extend these disjoint regions at least one generation of triangles deep into the interior of the cylinder to create quasicollars about the ends of the inserted cylinder. (Note that since the mesh of the packing is going to zero, the region occupied by one generation of triangles is getting arbitrarily small.) Since the inserted cylinder has non-zero height, we may construct these quasicollars so that they are disjoint.

Define a pullback map $P_{k}: \widehat{R}_{k} \rightarrow\left\{R_{k}, c_{1_{k}}, \ldots, c_{m_{k}}\right\}$ by letting $P_{k}(\hat{r})$ be the unique pre-image $r \in R_{k}$ of the point $\hat{r} \in \widehat{R}_{k}$ under the combinatorial earthquake $E_{k}$. Note that since the degree of the vertices in the complex on $\widehat{R}_{k}$ is bounded and since, by construction, the angles in the packing on $R_{k}$ are bounded away from zero, the map $P_{k}$ is $1+\hat{\varepsilon}_{k}$-quasiconformal. Further, at any point isolated from the combinatorial geodesics, as $k \rightarrow \infty$ the number of generations of circles on the packed surfaces between that point and the combinatorial geodesics goes to infinity; thus, at any such point, Lemma 3.7, the Packing Lemma, guarantees that the dilatation of the maps $P_{k}$ goes to 1 as $k \rightarrow \infty$, (i.e., as $k \rightarrow \infty$, on points isolated from the combinatorial geodesics, $P_{k} \rightarrow \widehat{P}$, where $\widehat{P}$ is conformal).

Since each of $R, R_{k}$, and $\widehat{R}_{k}$ are Riemann surfaces, they each have a conformal structure; in the case of $R_{k}$ and $\widehat{R}_{k}$, they inherit their conformal structures from their packings. Let $\left\{\varphi_{v}\right\}_{v \in \Upsilon},\left\{\psi_{\nu}\right\}_{\nu \in N},\left\{\varphi_{k_{v}}\right\}_{v \in \Upsilon_{k}}$, and $\left\{\psi_{k_{\nu}}\right\}_{k_{\nu} \in N_{k}}$ be the collection of coordinate charts in the conformal structure on $R, \widehat{R}, R_{k}$, and $\widehat{R}_{k}$, respectively. Note that the structure on $\widehat{R}$ will be the structure described in Section 2.7. Now, we consider a collection of maps $\hat{f}_{k}: \widehat{R}_{k} \rightarrow \widehat{R}$, each defined on open sets in $\widehat{R}_{k}$. Let $U \subset \widehat{R}_{k}$.

Case I: Suppose $U \subset \widehat{R}_{k}$ is an open set such that $U$ does not intersect the interior of any quasicollar, and $U$ does not intersect any cylinder inserted by the earthquake $E_{k}$. Associated with this open set in $\widehat{R}_{k}$ we have a coordinate chart $\psi_{k_{\nu}}$. Now, the map $P^{-1} \circ f_{k} \circ P_{k} \circ \psi_{k_{\nu}}^{-1}$ takes an open subset of the unit disk $\mathbf{D}$ corresponding to the pair $\left(U, \psi_{k_{\nu}}\right)$ to an open subset $U_{\alpha} \subset \widehat{R}$ so that $U_{\alpha} \in U_{A}$, as described in Section 2.7. Corresponding to $U_{\alpha}$, we take from the conformal structure on $\widehat{R}$ a coordinate chart $\psi_{\alpha}$. Thus we have

$$
\psi_{\alpha} \circ P^{-1} \circ f_{k} \circ P_{k} \circ \psi_{k_{\nu}}^{-1}=\varphi_{\alpha} \circ f_{k} \circ P_{k} \circ \psi_{k_{\nu}}^{-1} .
$$

Since $\varphi_{\alpha}$ and $\psi_{k_{\nu}}^{-1}$ are conformal (or 1-quasiconformal) and the maps $f_{k}$ and $P_{k}$ are $\left(1+\varepsilon_{k}\right)$-quasiconformal and $\left(1+\hat{\varepsilon}_{k}\right)$-quasiconformal, respectively, the map thus described is $\left(1+\varepsilon_{k}\right)\left(1+\hat{\varepsilon}_{k}\right)$-quasiconformal. We also know $\varepsilon_{k}$ and $\hat{\varepsilon}_{k}$ are independent of the set $U$, since they depend only on the maps $f_{k}$ and $P_{k}$, which are globally $\left(1+\varepsilon_{k}\right)$-quasiconformal and $\left(1+\hat{\varepsilon}_{k}\right)$-quasiconformal, respectively. By construction, $U$ is isolated in $\widehat{R}_{k}$ from the images of the geodesics defining the earthquake $E_{k}$, and $\varepsilon_{k}, \hat{\varepsilon}_{k} \rightarrow 0$ as $k \rightarrow \infty$. Thus, as $k \rightarrow \infty$, the map

$$
\psi_{\alpha} \circ P^{-1} \circ f_{k} \circ P_{k} \circ \varphi_{k_{\nu}}^{-1}: \widehat{R}_{k} \rightarrow \widehat{R}
$$

approaches a conformal map. 
Case II: Suppose $U \subset \widehat{R}_{k}$ is an open set such that $U$ does not intersect the interior of any quasicollar, and $U$ is contained in the interior of some cylinder inserted by the earthquake $E_{k}$. Associated with this open set in $\widehat{R}_{k}$ we have a coordinate chart $\psi_{k_{\nu}}$. Now, the map $P^{-1} \circ f_{j_{k}} \circ P_{k} \circ \psi_{k_{\nu}}^{-1}$ takes an open subset of the unit disk $\mathbf{D}$ corresponding to the coordinate pair $\left(U, \psi_{k_{\nu}}\right)$ from the conformal structure on $R_{k}$ to an open subset $U_{\beta} \subset \widehat{R}$ so that $U_{\beta} \in U_{B}$, as described in Section 2.7. Corresponding to $U_{\beta}$, we take from the conformal structure on $\widehat{R}$ described in Section 2.7 a coordinate chart $\psi_{\beta}$. Thus we have the following:

$$
\psi_{\beta} \circ P^{-1} \circ f_{j_{k}} \circ P_{k} \circ \psi_{k_{\nu}}^{-1}=M_{j} \circ g_{\beta}^{j} \circ \phi_{\beta}^{j} \circ f_{j_{k}} \circ P_{k} \circ \psi_{k_{\nu}}^{-1} .
$$

Since $M_{j} \circ g_{\beta}^{j} \circ \phi_{\beta}^{j}$ and $\psi_{k_{\nu}}^{-1}$ are conformal (or 1-quasiconformal) and the maps $f_{j_{k}}$ and $P_{k}$ are $\left(1+\varepsilon_{j_{k}}\right)$-quasiconformal and $\left(1+\hat{\varepsilon}_{k}\right)$-quasiconformal, respectively, the map thus described is $\left(1+\varepsilon_{j_{k}}\right)\left(1+\hat{\varepsilon}_{k}\right)$-quasiconformal. Again, we note that the constants describing the deviation from conformality, $\varepsilon_{j_{k}}$ and $\hat{\varepsilon}_{k}$, are independent of the choice of $U$. By construction, $U$ is isolated in $\widehat{R}_{k}$ from the images of the geodesics defining the earthquake $E_{k}$, and $\varepsilon_{j_{k}}, \hat{\varepsilon}_{k} \rightarrow 0$ as $k \rightarrow \infty$. Thus, as $k \rightarrow \infty$, the map $\psi_{\beta} \circ P^{-1} \circ f_{j_{k}} \circ P_{k} \circ \varphi_{k_{\nu}}^{-1}: \widehat{R}_{k} \rightarrow \widehat{R}$ approaches a conformal map.

Case III: Suppose that $U$ is a small open subset of $\widehat{R}_{k}$ such that $U$ intersects $E_{k}\left(\ell_{k}^{i}\right)$ for some $i=1,2, \ldots, n$, and $U$ intersects the boundary of the quasicollar $\widehat{C}_{k}^{i}$ associated with $L_{k}^{i}$. Corresponding to $U$ we have a coordinate chart $\psi_{k_{\nu}}$. Now, we have two cases to consider depending on whether the weight on the geodesic $L^{i}$ has non-zero imaginary part.

Case IIIa: Suppose $\operatorname{Im}\left(\sigma\left(L^{i}\right)\right)=0$; that is, the earthquake action on the geodesic $L^{i}$ involves only a shearing action. This gives that the map $P_{k}$ takes $U$ to a subset of $R_{k}$ such that $P_{k}(U) \nsubseteq C_{k}^{i}$ and $P_{k}(U) \bigcap \ell_{k}^{i} \neq \emptyset$.

We wish to send $P_{k}(U)$ to a subset of $R$, but there is a geometric difficulty to be resolved. The "halves" of $P_{k}(U)$ have as one portion of their boundaries a segment of the discrete geodesic $\ell_{k}^{i}$. We need these segments of their boundaries to lie on the actual geodesic $L_{k}^{i}$ in $R_{k}$. The problem is easily resolved, at the expense of admitting some quasiconformality in our eventual transformation, by the application of the hyperbolic projection $p_{k}^{i}$ described in Section 4.2, as illustrated in Figure 5.

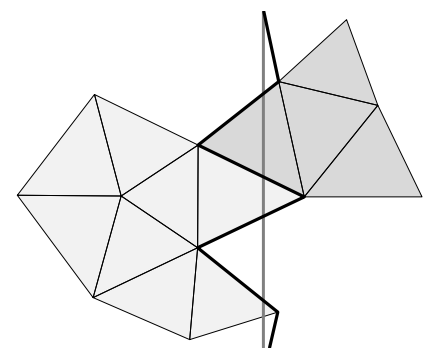

(a) Result of the pullback $P_{k}(U)$.

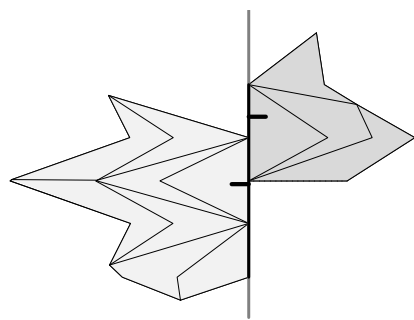

(b) Result of the projection $p_{k}^{i}\left(P_{k}(U)\right)$.

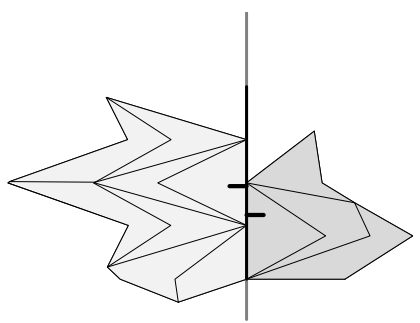

(c) Post-shearing misalignment.

Figure 5. Projection of geodesics, discrete geodesics, and quasicollars and a misalignment resulting from the application of a shearing map.

The map $p_{k}^{i}$ on $P_{k}(U)$ is $1+\tilde{\varepsilon}_{k}$-quasiconformal, but Proposition 4.2 guarantees that $p_{k}^{i}$ converges uniformly to the identity; so $\tilde{\varepsilon}_{k} \rightarrow 0$ as $k \rightarrow \infty$. Further, the 
constant $\tilde{\varepsilon}_{k}$ is independent of the choice of $U$. This independence is a result of two facts. First, the projection associated with any given geodesic $L^{i}$ and the accompanying discrete geodesic $\ell_{k}^{i}$ has a quasiconformality constant uniform across the entire geodesic. Second, there are only finitely many geodesics for which we must use these projections. Taking the maximum such quasiconformal constant as $\tilde{\varepsilon}_{k}$, we have independence of the choice for $U$.

Corresponding to the set $p_{k}^{i}\left(P_{k}(U)\right) \subset R_{k}$, or more precisely to some open subset of $R_{k}$ containing $p_{k}^{i}\left(P_{k}(U)\right) \subset R_{k}$, we have a coordinate chart $\varphi_{k_{v}}$ which takes $p_{k}^{i}\left(P_{k}(U)\right)$ to a subset of a fundamental region for $R_{k}$ in the Poincaré unit disk, D. The image of $p_{k}^{i}\left(P_{k}(U)\right) \bigcap L_{k}^{i}$ under this map is thus an arc of a hyperbolic geodesic in $\mathbf{D}$. There exists a disk automorphism $M_{k}^{U}: \mathbf{D} \rightarrow \mathbf{D}$ such that $\left.M_{k}^{U}\left(\varphi_{k_{v}}\left(p_{k}^{i}\left(P_{k}(U)\right)\right)\right) \bigcap L_{k}^{i}\right)$ is a small arc of a hyperbolic geodesic in $\mathbf{D}$ that is the projection of the geodesic $L^{i}$ in $R$. We may choose this arc of the projection of $L^{i}$ sufficiently small that the set $M_{k}^{U}\left(\varphi_{k_{v}}\left(p_{k}^{i}\left(P_{k}(U)\right)\right)\right)$ is contained in a single fundamental region for $R$.

A second difficulty arising from the projection operation must be addressed. If we were to apply a shearing map on the lift of the geodesic $L^{i}$ which separates the "halves" of $M_{k}^{U}\left(\varphi_{k_{v}}\left(p_{k}^{i}\left(P_{k}(U)\right)\right)\right)$ using the weight associated with $L^{i}$, the sides will not necessarily match along the geodesic, as illustrated in Figure 5.

The difference along the geodesic is a quasisymmetry on one half of the intersection with $L^{i}$, however, and extends to a $1+\bar{\varepsilon}_{k}$-quasiconformal map, $Q_{k}^{U}$, in the corresponding half of $M_{k}^{U}\left(\varphi_{k_{v}}\left(p_{k}^{i}\left(P_{k}(U)\right)\right)\right)$ [14, 19]. Recall, however, that the quasisymmetry and associated quasiconformal map arise as a result of the need for a hyperbolic projection $p_{k}^{i}$ to correct the difference between the geodesic $L_{k}^{i}$ and the discrete geodesic $\ell_{k}^{i}$. Lemma 4.1 guarantees that $\ell_{k}^{i} \rightarrow L_{k}^{i}$ uniformly as $k \rightarrow \infty$. Thus, $\bar{\varepsilon}_{k} \rightarrow 0$ as $k \rightarrow \infty$. Further, since the quasiconformality of $M_{k}^{U}$ depends only on the quasisymmetry associated with the projection $p_{k}^{i}, \bar{\varepsilon}_{k}$ is independent of the choice of $U$.

Corresponding to the set $Q_{k}^{U}\left(M_{k}^{U}\left(\varphi_{k_{v}}\left(p_{k}^{i}\left(P_{k}(U)\right)\right)\right)\right) \subset \mathbf{D}$, we have a map from the conformal structure on $R, \varphi_{v}^{-1}$, taking this set to an open set on $R$ intersecting the geodesic $L^{i}$. (Again, the coordinate chart really comes from an open set containing the set of interest.) Notice that as a result of applying the correction $Q_{k}^{U}$, the two halves of this image (on either side of the lift to $\mathbf{D}$ of the geodesic $L^{i}$ ) now match the action of the shearing caused by $P^{-1}$. We now apply $P^{-1}$, to obtain a set $V \subset \widehat{R}$. Corresponding to this open set we have a coordinate chart from the conformal structure on $\widehat{R}$ defined in Section 2.7. Since $V \in U_{\Gamma}$, we have a coordinate chart $\psi_{\gamma}$ corresponding to $V$. This gives the following:

$$
S_{\gamma} \circ \varphi_{\gamma} \circ \varphi_{v}^{-1} \circ Q_{k}^{U} \circ M_{k}^{U} \circ \varphi_{k_{v}} \circ p_{k}^{i} \circ P_{k} \circ \varphi_{k_{\nu}}^{-1}
$$

This map is $\left(1+\bar{\varepsilon}_{k}\right)\left(1+\tilde{\varepsilon}_{k}\right)\left(1+\hat{\varepsilon}_{k}\right)$-quasiconformal, where this quasiconformality is independent of the choice for $U$. Now, consider the dilatation at a point $u \in U$. If $u$ is not contained in the set $E_{k}\left(\ell_{k}^{i}\right)$, then Lemma 3.7, the packing Lemma, guarantees that the dilatation at $u$ goes to 1 since each of $\bar{\varepsilon}_{k}, \tilde{\varepsilon}_{k}, \hat{\varepsilon}_{k} \rightarrow 0$ as $k \rightarrow \infty$. Thus, this map from $\widehat{R}_{k}$ to $\widehat{R}$ converges to a conformal map, except perhaps on the image of the discrete geodesic $\ell_{k}^{i}$ under the earthquake $E_{k}$. But this set has measure zero, and the map is continuous. Thus, the limit map is conformal on all of $U$ [19]. 
Case IIIb: Suppose $\operatorname{Im}\left(\sigma\left(L^{i}\right)\right)>0$; that is, the earthquake action on the geodesic $L^{i}$ is a grafting action (and potentially a shearing action as well). The situation here is somewhat more complicated than the previous case since the map $P_{k}$ takes the portion of $U$ on the inserted cylinder to a cylinder $c_{j_{k}}$ and the remaining points of $U$ to a subset of $R_{k}$. We will deal with the maps on these two subsets of $U$ separately.

First, consider that subset of $U$ which is taken to $R_{k}$. Call this subset $U_{1}$. $P_{k}\left(U_{1}\right) \nsubseteq C_{k}^{i}$ and $P_{k}\left(U_{1}\right) \bigcap \ell_{k}^{i} \neq \emptyset$. We wish to send this set $P_{k}\left(U_{1}\right)$ to a subset of $R$, but there is a geometric difficulty to be resolved. $P_{k}\left(U_{1}\right)$ has as one portion of its boundary a segment of the discrete geodesic $\ell_{k}^{i}$; we need this segment of the boundary to lie on the actual geodesic $L_{k}^{i}$ in $R_{k}$. As before, this problem is easily addressed, at the expense of admitting some quasiconformality into our eventual transformation, by the application of the hyperbolic projection $p_{k}^{i}$ described in Section 4.2. This map on the set $P_{k}\left(U_{1}\right)$ is $1+\tilde{\varepsilon}_{k}$-quasiconformal, but Proposition 4.2 guarantees that $p_{k}^{i}$ converges uniformly to the identity; so $\tilde{\varepsilon}_{k} \rightarrow 0$ as $k \rightarrow \infty$. As in Case IIIa, $\tilde{\varepsilon}_{k}$ is independent of the choice of $U$.

Corresponding to the set $p_{k}^{i}\left(P_{k}\left(U_{1}\right)\right) \subset R_{k}$, or more precisely to some open subset of $R_{k}$ containing $p_{k}^{i}\left(P_{k}\left(U_{1}\right)\right) \subset R_{k}$, we have a coordinate chart $\varphi_{k_{v}}$ which takes $p_{k}^{i}\left(P_{k}\left(U_{1}\right)\right)$ to a subset of a fundamental region for $R_{k}$ in the Poincaré unit disk, D. The image of $p_{k}^{i}\left(P_{k}\left(U_{1}\right)\right) \cap L_{k}^{i}$ under this map is thus an arc of a hyperbolic geodesic in $\mathbf{D}$. There exists a disk automorphism $M_{k}^{U}: \mathbf{D} \rightarrow \mathbf{D}$ such that $\left.M_{k}^{U}\left(\varphi_{k_{v}}\left(p_{k}^{i}\left(P_{k}\left(U_{1}\right)\right)\right)\right) \bigcap L_{k}^{i}\right)$ is a small arc of the hyperbolic geodesic in $\mathbf{D}$ that results from the lift of the geodesic $L^{i}$ in $R$. We may choose $M_{k}^{U}$ such that the set $M_{k}^{U}\left(\varphi_{k_{v}}\left(p_{k}^{i}\left(P_{k}\left(U_{1}\right)\right)\right)\right)$ is an open set intersecting this geodesic in a fundamental region for $R$.

Again, we will require a correction to compensate for the effect of the hyperbolic projection $p_{k}^{i}$. Call this correction $Q_{k}^{U}$, and note that this map is a quasisymmetry on the intersection with the lift of the geodesic $L^{i}$ and is $\left(1+\tilde{\varepsilon}_{k}\right)$-quasiconformal, as in Case IIIa. Again, the quasiconformality constant $\bar{\varepsilon}_{k}$ is independent of the choice for $U$. Corresponding to the set $Q_{k}^{U}\left(M_{k}^{U}\left(\varphi_{k_{v}}\left(p_{k}^{i}\left(P_{k}\left(U_{1}\right)\right)\right)\right) \subset \mathbf{D}\right.$, we have a map from the conformal structure on $R, \varphi_{v}^{-1}$, taking this set to an open set on $R$ intersecting the geodesic $L^{i}$. We now apply $P^{-1}$, to obtain a set $V_{1} \subset \widehat{R}$ adjoining the edge of an inserted cylinder $c_{j}$.

Now, consider that subset of $U$ which is taken into $c_{j_{k}}$. Call this subset $U_{2}$. $P_{k}\left(U_{2}\right) \subset c_{j_{k}}$ and $P_{k}\left(U_{2}\right)$ intersects the boundary of the cylinder $c_{j_{k}}$. We take this set into the cylinder $c_{j}$ via the $1+\tilde{\epsilon}_{k}$-quasiconformal map $f_{j_{k}}$, where $\tilde{\epsilon}_{k}$ is independent of the choice of $U$, since we may choose $\tilde{\epsilon}_{k}$ as the maximum of the constants associated with the finitely many cylinders $\left\{c_{j}\right\}$. We then apply the map $P^{-1}$ to obtain a set $V_{2} \subset \widehat{R}$.

The way we have constructed these maps, in particular the application of the map $p_{k}^{i}$, ensures that the set $V=V_{1} \bigcup V_{2} \subset \widehat{R}$ is an element of the collection $U_{\Delta}$ as described in Section 2.7. Corresponding to this set $V$ we have a coordinate chart $\psi_{\delta}$ from the conformal structure on $\widehat{R}$. This gives a piecewise map $\Pi_{k}: \widehat{R}_{k} \rightarrow \widehat{R}$ defined by

$$
\Pi_{k}(u)=\left\{\begin{array}{l}
\psi_{\delta} \circ P^{-1} \circ \varphi_{v}^{-1} \circ Q_{k}^{U} \circ M_{k}^{U} \circ \varphi_{k_{v}} \circ p_{k}^{i} \circ P_{k} \circ \psi_{k_{\nu}}^{-1}(u), \\
\psi_{\delta} \circ P^{-1} \circ f_{j_{k}} \circ P_{k} \circ \psi_{k_{\nu}}^{-1}(u) .
\end{array}\right.
$$


The first of these maps, for $u \in U_{1}$, is $\left(1+\bar{\varepsilon}_{k}\right)\left(1+\tilde{\varepsilon}_{k}\right)\left(1+\hat{\varepsilon}_{k}\right)$-quasiconformal. The second map, for $u \in U_{2}$, is $\left(1+\varepsilon_{j_{k}}\right)\left(1+\hat{\varepsilon}_{k}\right)$-quasiconformal. As we have already shown, each of these constants is independent of the choice of $U$. Now, consider the dilatation at a point $u \in U$. If $u$ is not contained in the set $E_{k}\left(\ell_{k}^{i}\right)$, then the Packing Lemma guarantees the dilatation at $u$ goes to 1 since each of $\bar{\varepsilon}_{k}, \tilde{\varepsilon}_{k}, \hat{\varepsilon}_{k}, \varepsilon_{j_{k}} \rightarrow 0$ as $k \rightarrow \infty$. Thus, this piecewise map $\Pi_{k}$ converges to a conformal map $\Pi$, except perhaps on the image of the discrete geodesic $\ell_{k}^{i}$ under the earthquake $E_{k}$. But this set has measure zero, and the map is continuous. Thus, the map $\Pi$ is conformal on all of $U[19]$.

Thus, as $k \rightarrow \infty$ the maps from $\widehat{R}_{k}$ to $\widehat{R}$ are becoming conformal, and, by construction, the markings on surfaces $\widehat{R}$ and $\widehat{R}_{k}$ are consistent. Therefore, the surfaces $\widehat{R}_{k}$ converge to a surface conformally equivalent to $\widehat{R}$ with equivalent markings; but this is equivalent to saying $\widehat{R}_{k} \rightarrow \widehat{R}$ (in the Teichmüller metric) as $k \rightarrow \infty$, and the result is shown.

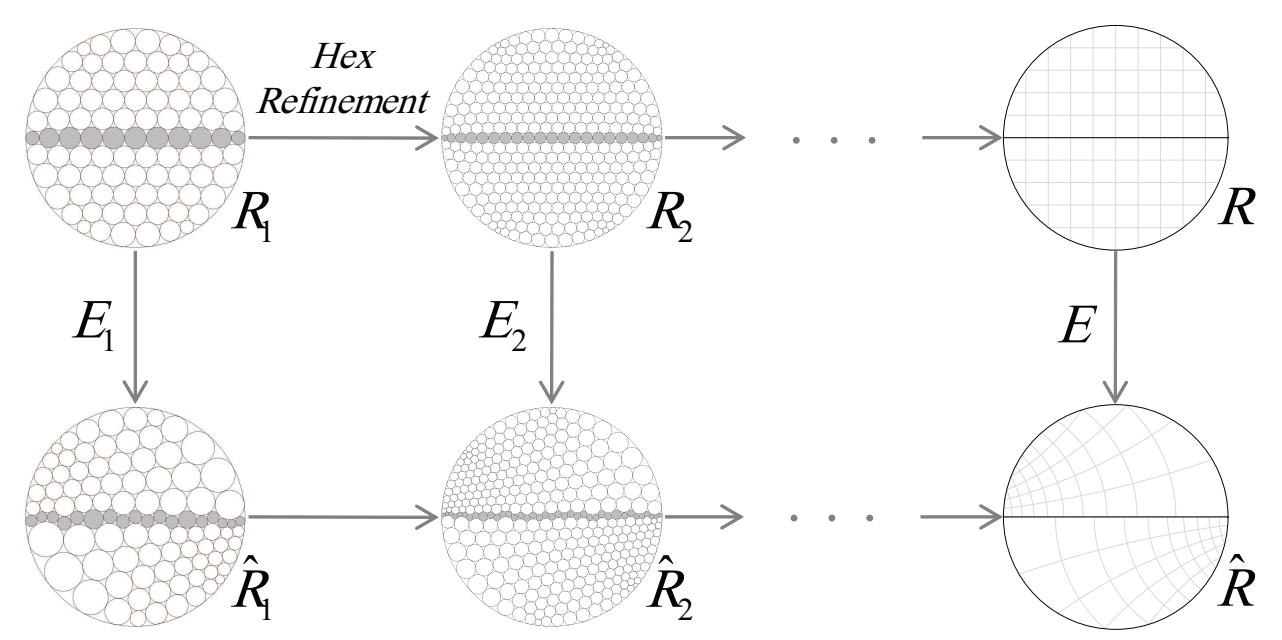

Figure 6. A sequence of discrete earthquake maps approximating a shearing action on the Poincare disk model of the hyperbolic plane.

We provide two illustrative examples of earthquakes in the Poincaré unit disk in Figure 6 and Figure 7. In each, we approximate the unit disk with a succession of hex refinements of a regular hexagonal packing (top) and execute a discrete conformal approximation of an earthquake along the geodesic given by the real axis. In these examples, the discrete geodesic coincides with the real axis, illustrated by the circles corresponding to this geodesic shown in gray.

In Figure 6 we simultaneously execute a left shearing map on the upper and lower half-disks (as seen from the opposite half of the unit disk), where each of these actions has a hyperbolic weight $\mu=1$. The action of these discrete conformal shearing actions is suggested by the images $\widehat{R}_{1}$ and $\widehat{R}_{2}$, and the expected convergence to $\widehat{R}$ is illustrated (where the image of the disk under the explicit shearing map $\widehat{R}$ is shown). In Figure 7 we perform a hyperbolic graft in which a strip of hyperbolic width $\lambda=1$ is inserted along the real axis. As before, the expected convergence to the surface $\widehat{R}$ is clearly illustrated by the discrete conformal action of the maps $E_{1}$, $E_{2}$, and $E_{3}$. The inserted cylinder is shown in gray. 


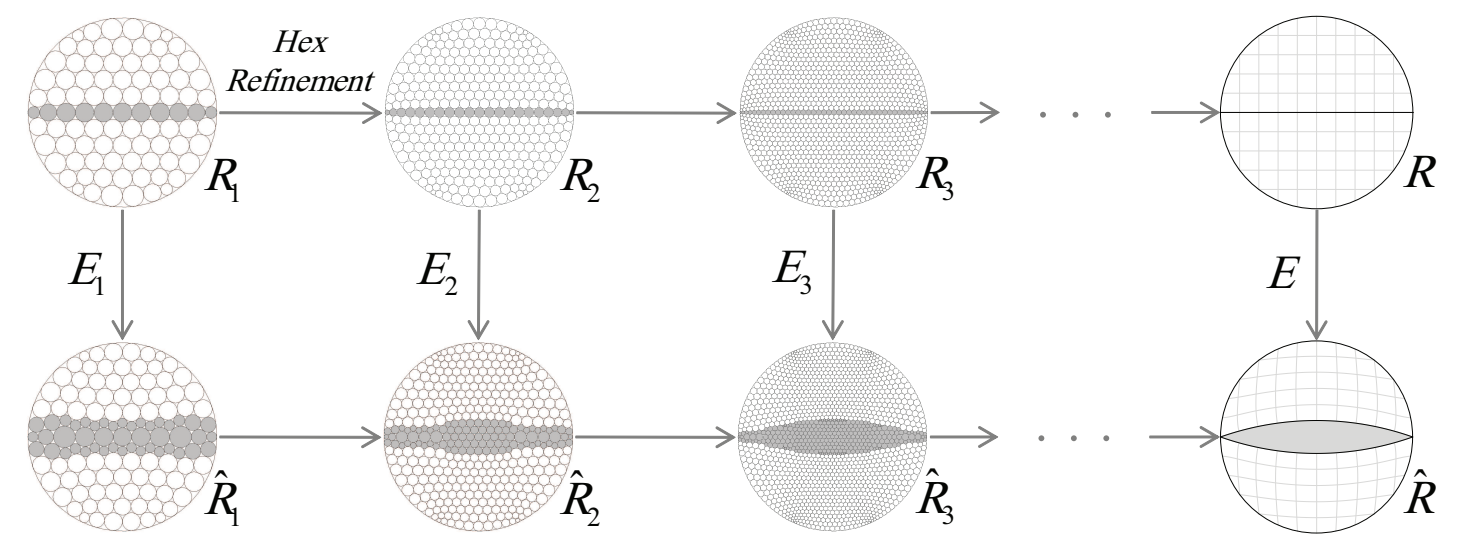

Figure 7. A sequence of discrete earthquake maps approximating a grafting action on the Poincaré disk model of the hyperbolic plane.

A similar result holds for circle packing approximations of complex earthquakes on compact tori. These earthquakes are described fully in [2], and an argument parallel to that given above yields the following result.

Theorem 4.5. Let $T$ be a compact Euclidean Riemann surface of genus $g=1$ (a torus) with a finite measured geodesic lamination $(\mathcal{L}, \sigma)$. Let $\left\{P_{k}\right\}$ be a sequence of finite bounded degree packings with mesh decreasing to zero corresponding to Riemann surfaces $\left\{T_{k}\right\}$ such that $T_{k} \rightarrow T$ as $k \rightarrow \infty$ in the Teichmüller metric. Then the surfaces $E_{k}\left(T_{k}\right)=\widehat{T}_{k}$ induced by the discrete Euclidean earthquake maps $E_{k}$ converge to the surface $E(T)=\widehat{T}$ under the Euclidean earthquake map $E$ induced by $(\mathcal{L}, \sigma)$.

\section{References}

[1] Barnard, R. W., E. M. Murphy, and G. B. Williams: Some results on spaces of packable Riemann surfaces. - J. Anal. 15, 2007, 1-15.

[2] Barnard, R. W., E. M. Murphy, and G. B. Williams: Euclidean earthquakes. - Complex Var. Elliptic Equ. 54:7, 2009, 639-652.

[3] Barnard, R. W., and G. B. Williams: Combinatorial excursions in moduli space. - Pacific J. Math. 205:1, 2002, 3-30.

[4] Bely̆̆, G.: On Galois extensions of a maximal cyclotomic field. - Math. USSR Izv. 14:2, 1980, 247-256 (in English).

[5] Beardon, A.F., and K. Stephenson: The uniformization theorem for circle packings. Indiana Univ. Math. J. 39, 1990, 1383-1425.

[6] Bonahon, F.: Earthquakes on Riemann surfaces and on measured geodesic laminations. Trans. Amer. Math. Soc. 330:1, 1992, 69-95.

[7] Bowers, P. L., and K. Stephenson: Circle packings in surfaces of finite type: An in situ approach with application to moduli space. - Topology 32:1, 1993, 157-183.

[8] Bowers, P. L., and K. Stephenson: The set of circle packing points in the Teichmüller space of a surface of finite conformal type is dense. - Math. Proc. Cambridge Philos. Soc. 111:3, 1992, $487-513$.

[9] Bowers, P. L., and K. Stephenson: Uniformizing Dessins and Belyŭ maps via circle packing. - Mem. Amer. Math. Soc., Amer. Math. Soc., 2004.

[10] Brooks, R.: Circle packings and co-compact extensions of Kleinian groups. - Invent. Math. $86: 3,1986,461-469$. 
[11] Buser, P.: Geometry and spectra of compact Riemann surfaces. - Birkhäuser, Boston, 1992.

[12] Carathéodory, C.: Conformal representation. - Dover, New York, 1932.

[13] Dubejko, T., and K. Stephenson: Circle packing: Experiments in discrete analytic function theory. - Experiment. Math. 4:4, 1995, 307-348.

[14] Gardiner, F.P., and N. Lakic: Quasiconformal Teichmüller theory. - Math. Surveys Monogr. 76, Amer. Math. Soc., 2000.

[15] He, Z.-X., and B. Rodin: Convergence of circle packings of finite valence to Riemann mappings. - Comm. Anal. Geom. 1, 1993, 31-41.

[16] Imayoshi, Y., and M. Taniguchi: An introduction to Teichmüller spaces. - Springer-Verlag, New York, 1992.

[17] Jones, G. A., and D. Singerman: Complex functions: An algebraic and geometric viewpoint. - Cambridge Univ. Press, Cambridge, 1987.

[18] Kerckhoff, S. P.: The Nielsen realization problem. - Ann. of Math. (2) 117, 1983, 235-265.

[19] Lehto, O., and K. I. Virtanen: Quasiconformal mappings of the plane, 2nd edition. Springer-Verlag, New York, 1973.

[20] McMullen, C. T.: Complex earthquakes and Teichmüller theory. - J. Amer. Math. Soc. 11:2, 2001, 283-320.

[21] Minda, D., and B. Rodin: Circle packing and Riemann surfaces. - J. Anal. Math. 57:1, 1991, $221-249$.

[22] Rodin, B., and D. Sullivan: The convergence of circle cackings to the Riemann mapping. J. Differential Geom. 26:2, 1987, 349-360.

[23] Stephenson, K.: An introduction to circle packing: The theory of discrete analytic functions. - Cambridge Univ. Press, 2005.

[24] Stephenson, K.: A probabalistic proof of Thurston's conjecture on circle packings. - Rend. Sem. Math. Fis. Milano 66, 1996, 201-291.

[25] Stephenson, K.: Circle packing: A mathematical tale. - Notices Amer. Math. Soc. 50:11, 2003, 1376-1388.

[26] Stephenson, K.: Circle packing and discrete analytic function theory. - In: Handbook of complex analysis, volume 1: Geometric function theory, edited by R. Kühnau, Elsevier, 2002, $333-370$.

[27] Stillwell, J.: Geometry of surfaces. - Springer-Verlag, New York, 1992.

[28] Thurston, W. P.: Earthquakes in two-dmensional hyperbolic geometry. - In: Lowdimensional topology and Kleinian groups (Coventry/Durham, 1984), London Math. Soc. Lecture Note Ser. 112, Cambridge Univ. Press, 1986, 90-112.

[29] Thurston, W. P.: The Finite Riemann Mapping Theorem. - Invited talk, An International Symposium at Purdue University on the occasion of the proof of the Bieberbach conjecture, 1985.

[30] Thurston, W.P.: The geometry and topology of 3-manifolds. - Princeton Univ. Notes, preprint.

[31] Williams, G. B.: Discrete approximations of conformal weldings using circle packing. - Indiana Univ. Math. J. 53:3, 2004, 765-804.

[32] Williams, G. B.: Earthquakes and circle packing. - J. Anal. Math. 85:1, 2001, 371-396. 